\title{
Influence of obesity on the outcome of non-surgical periodontal therapy - a systematic review
}

\author{
Fabienne A. Gerber ${ }^{1}$, Philipp Sahrmann¹, Oliver A. Schmidlin², Christian Heumann³, Jürg Hans Beer² \\ and Patrick R. Schmidlin ${ }^{1 *}$
}

\begin{abstract}
Background: Obesity and periodontitis are important chronic health problems. Obesity is associated with an increased prevalence of periodontitis. Whether obesity also affects the outcome of non-surgical periodontal therapy is to date still unclear.

Methods: A systematic review of studies referenced in SCOPUS, MEDLINE, PubMed, Cochrane, CINAHL, Biosis and Web of Science was performed. Titles, abstracts and finally full texts were scrutinized for possible inclusion by two independent investigators. Quality and heterogeneity of the studies were assessed and the study designs were examined. Probing pocket depth reduction was analyzed as primary surrogate parameter for therapeutic success after non-surgical periodontal therapy.

Results: One-hundred-and-fifty-nine potentially qualifying studies were screened. Eight studies fulfilled the inclusion criteria and were analyzed. Three of eight studies failed to show an influence of obesity on pocket depth reduction after non-surgical therapy. The remaining five studies documented a clear negative effect on the outcome of non-surgical periodontal therapy. The finally included studies did not correspond to the highest level of quality (RCTs). Due to the heterogeneity of the data a meta-analysis was not possible.

Conclusion: The literature on the effect of obesity on the treatment outcome of non-surgical periodontal therapy remains controversial. The data, however, support that obesity is not only a factor associated with poorer periodontal health but might also result in inferior response to non-surgical treatment of periodontitis.
\end{abstract}

Keywords: Obesity, Chronic periodontitis, Non-surgical periodontal therapy, Outcome

\section{Background}

The prevalence of obesity is increasing worldwide and is becoming one of the most important health hazards [1], as obesity is highly associated with increased overall morbidity and mortality [2].

Obesity is defined with a body mass index (BMI; body weight in kilogram divided by the square of the height in meters $\left.\left(\mathrm{kg} / \mathrm{m}^{2}\right)\right)$ of at least $30.0 \mathrm{~kg} / \mathrm{m}^{2}$ [3], whereas overweight is defined with a BMI of $25-29.9 \mathrm{~kg} / \mathrm{m}^{2}$. Normal weight is characterized by a BMI ranging between 19 to

\footnotetext{
* Correspondence: patrick.schmidlin@zzm.uzh.ch

${ }^{1}$ Clinic of Preventive Dentistry, Periodontology and Cariology, Center of Dental Medicine, University of Zurich, Plattenstrasse 11, CH-8032 Zurich, Switzerland

Full list of author information is available at the end of the article
}

$24.9 \mathrm{~kg} / \mathrm{m}^{2}$ [4]. In this context, BMI seems a valuable parameter to predict obesity-related disease risks in a wide range of populations [2]. There are, however, some limitations: Firstly, risk assessment by BMI is less applicable in persons over 65 years of age because they generally have a higher body fat content for the same BMI. Secondly, the abdominal (central, visceral, android) type of obesity, which is more often seen in men, is associated with higher morbidity than the rather female type of gluteofemoral (peripheral, gynoid) obesity and, thirdly, the BMI cut-off points for overweight and obesity are too high for Asian people [2]. In addition, current large studies have indicated that measurement of waist circumference (WC) or waist-to-hip-ratio (WHR) may be a better disease risk predictor than BMI $[5,6]$. There is, 
however, currently intensive research and debate as to whether BMI, WC, WHR, or all of them should be used to assess disease risk [2].

For the purpose of this systematic review, however, only BMI is the most frequently reported data of obesity in a large number of studies.

Adipose tissue contains usually 5-10 \% macrophages, but the adipose tissue of obese patients shows up to $60 \%$ macrophage infiltration [4]. Adipocytes secrete bioactive molecules called adipokines, that can modify or trigger inflammation and fat metabolism locally or systemically as signaling molecules to liver, muscle and endothelium [4]. Therefore, the adipose tissue can be considered as an important metabolically active endocrine organ [4].

This explains how obesity acts as a risk factor for several chronic diseases: Hypertension, type 2 diabetes, dyslipidemia, and coronary heart disease are so closely related to obesity that obesity itself is often considered to be a systemic disease. This disease also affects dental health [7]. Accordingly obese persons require attention of physicians and dentists [2].

Among dental pathologies, periodontitis is a very common, primarily bacterial inflammatory disease, which destroys teeth surrounding soft tissues and bone. It leads to pocket formation and ultimately to loss of teeth if no effective treatment is applied [8]. Periodontitis is no longer considered only an oral health issue but also a public health problem, as it constitutes a risk factor for cardiovascular conditions, poor glycemic control in diabetics and adverse outcome of pregnancy $[4,8]$. These correlations coincide with obesity and general health.

Recently, it has been suggested that obesity is a possible risk factor for periodontitis [8]. One study identified obesity even as the second strongest risk factor for periodontitis preceded only by smoking [9]. The first report on the relationship between obesity and periodontal disease appeared in 1977. Perlstein and co-workers [10] found greater alveolar bone resorption in obese than in non-obese rats. Under healthy oral conditions, obesity itself did not promote periodontal damage, but in the presence of bacterial plaque accumulation periodontal inflammation was more severe in obese than in nonobese animals. With concomitant arterial hypertension, plaque accumulation caused even more pronounced periodontal destruction than with obesity alone. These results suggest that a combination of risk factors, such as the one defined by the metabolic syndrome, elicit a more severe periodontal effect $[10,11]$. Chaffee et al. [12] found in their meta-analysis an increased prevalence odds ratio for obesity among subjects with periodontal disease of approximately one-third, a greater mean clinical attachment loss (CAL) among obese individuals, a higher BMI among subjects with periodontal disease, and a trend for linear increase in the odds of periodontal disease with increasing BMI $[4,12]$. Finally the association reported between obesity and periodontitis was less strong than that reported between periodontal disease and adverse pregnancy outcomes $[12,13]$ or cardiovascular events $[12,14]$. There seems, however, to be a stronger obesity-periodontitis association in women, non-smokers and younger individuals than in the general adult populations [12]. In addition, smoking remains another well-studied predisposing factor for periodontitis $[12,15,16]$. Thus, BMI and smoking share a complex relationship [17]. This relationship can be inverse in certain populations $[12,18,19]$.

The biological mechanism by which obesity predisposes to periodontitis is not fully understood [8]. Compared to individuals with normal weight individuals with obesity have higher levels of circulating tumor necrosis factor- $\alpha$ (TNF- $\alpha$ ) and interleukin-6 (IL-6), which are also secreted from adipose tissue and are involved in the pathophysiology of both obesity and periodontitis. Not surprisingly, serum levels of these cytokines decrease with loss of weight [20].

The objective of this systematic review was to study the hypothesis whether the clinical outcome, in terms of pocket depth reduction, after non-surgical periodontal therapy in non-obese is better than in obese individuals. To verify this hypothesis, we systematically reviewed all retrievable, qualitatively adequate clinical investigations, which focused on this topic.

\section{Methods}

The review was conducted according to the PRISMA criteria [21]. The research question was explored using the PICO method [22). The focused question addressed was:

Does non-surgical periodontal therapy (I) have a different outcome in obese chronic periodontitis patients $(\mathrm{P})$, than in non-obese chronic periodontitis patients (C), regarding periodontal pocket depth reduction as the main clinical periodontal parameter $(\mathrm{O})$.

\section{Search strategy and review process}

An electronic search of SCOPUS, MEDLINE, PubMed, Cochrane, CINAHL, Biosis and Web of Science was carried out considering articles published up to January 2016 in English or German language. The search was performed in two steps. The first electronic search started at 20.11.14 and an update has been done at 5.1.16.

This is shown in Table 1. For the database search, a combination of subject headings (MeSH terms and CINAHL headings) and free text search was used. An example of a detailed strategy (Medline/OvidSP) is shown in Table 2.

The same search protocol was applied to all databases. 
Table 1 Search protocols with the respective number of references dated from November 112014 (1) and January 1 $2016(2)$

\begin{tabular}{lll}
\hline & References $1 \& 2$ & References after deduplication 1 \& 2 \\
\hline Scopus & $40+14$ & $9+5$ \\
Medline & $42+8$ & $39+7$ \\
PubMed & $6+11$ & $2+11$ \\
Cochrane & $12+2$ & $2+1$ \\
CINAHL & $48+15$ & $42+13$ \\
Biosis & $25+7$ & $10+1$ \\
Web of Science & $38+13$ & $16+1$ \\
Pool & $211+70$ & $120+39$ \\
\hline
\end{tabular}

After deduplication 159 references

Two of the authors (FAG, PRS) screened the titles for potential eligibility according to the inclusion criteria. Based on the abstract screening, 18 studies were selected for full text review. Scores were independently allocated by both authors to each publication according to their

Table 2 Medline search strategy

\begin{tabular}{|c|c|c|}
\hline Step & Query & Hits \\
\hline 1 & Exp Periodontitis/AND (adult OR chronic*).ti,ab. & 5703 \\
\hline 2 & Chronic periodontitis/OR periodontal pocket/ & 7004 \\
\hline 3 & 2 OR 3 & 10454 \\
\hline 4 & ((adult OR chronic*) adj3 periodontiti*).ti,ab. & 5352 \\
\hline 5 & $\begin{array}{l}\text { (attachment adj3 loss).ti,ab. AND ((clinical OR } \\
\text { periodontal) adj3 attachment).ti,ab. }\end{array}$ & 1305 \\
\hline 6 & (periodontal adj3 pocket).ti,ab. & 1066 \\
\hline 7 & OR/3-7 & 12910 \\
\hline 8 & Dental scaling/ OR "root planing"/ & 3479 \\
\hline 9 & $\begin{array}{l}\text { ((subgingival OR root OR supragingival) adj3 } \\
\text { (scaling* OR planing*)).ti,ab. }\end{array}$ & 2288 \\
\hline 10 & (deep adj3 (scaling* OR cleaning)).ti,ab. & 62 \\
\hline 11 & $\begin{array}{l}\text { ((conventional OR inital OR “non surgical” OR } \\
\text { non-surgical OR nonsurgical) adj3 } \\
\text { (treatment OR therapy)).ti,ab. }\end{array}$ & 33417 \\
\hline 12 & OR/8-11 & 37131 \\
\hline 13 & 7 AND 12 & 2184 \\
\hline 14 & Exp Obesity/ & 154540 \\
\hline 15 & Exp Body Weight/ & 369850 \\
\hline 16 & $\begin{array}{l}\text { (overweight OR obese OR obesity OR adiposit* } \\
\text { OR BMI OR "body mass index").ti,ab. }\end{array}$ & 300847 \\
\hline 17 & (cardiolipin OR lipid* OR leptin).ti,ab. & 395517 \\
\hline 18 & "waist to hip".ti,ab. & 9048 \\
\hline 19 & (body adj3 weight).ti,ab. & 163760 \\
\hline 20 & OR/14-19 & 954233 \\
\hline 21 & 13 AND 20 & 42 \\
\hline
\end{tabular}

The asterisk represents a wildcard and can be used in all search fields that allow words and phrases suitability for the present review (see inclusion criteria). Any discrepancies were resolved by consensus.

\section{Inclusion criteria}

To be included studies had to be clinical interventional studies regarding the outcome of non-surgical periodontal therapy in obese or non-obese patients. The studies had to display the diagnosis of chronic periodontitis. Key parameters to be reported were data for pocket probing depth (PPD) and BMI.

\section{Exclusion criteria}

Studies were excluded for the following reasons: animal studies, case reports, commentaries, unsuitable exposure or outcome measures, confounding medical diagnoses (e.g. pregnancy or any systemic disease, such as diabetes, in addition to metabolic syndrome), confounding systemic medical treatments such as immunosuppressive treatments, cortisone or antibiotic treatment as well as confounding local treatments such as treatment of perimucositis, gingival overgrowth or surgical periodontal treatment. Studies including either the diagnosis of aggressive periodontitis or of peri-implantitis were excluded as well.

\section{Outcome measures}

The primary outcome measure is PPD after non-surgical periodontal treatment.

\section{Data extraction}

A list with exclusion reasons for each paper was generated. Total number of patients, demographic data, origin of study, outcome measurements 2, 3, 6 and 12 months after therapy and the impact of obesity on the treatmentoutcome were extracted. In addition, the exact definition of chronic periodontitis, the assessment of the periodontal disease and the number of smokers included in the studies were summarized. Data on the individual definition of obesity and the systemic examinations were also collected. Nonsurgical periodontal treatment measures, treatment time, periodontal maintenance and adverse events were also recorded for each study separately (Tables 3, 4, 5, 6 and 7).

The quality of the included studies was assessed through the Newcastle-Ottawa Quality Assessment Scale (Table 8).

\section{Results}

\section{Selection of studies}

Initially, 159 studies were identified by electronic search by the two reviewers (FAG, PRS). Full text analysis of the 18 potentially qualified reports led to exclusion of 10 other studies. Additional five titles [23-27] were identified by hand search but after full text analysis, all these articles had to be excluded based on the inclusion and exclusion 
Table 3 Excluded studies

\begin{tabular}{l}
\hline Excluded studies \\
\hline Europerio 42003 [37) \\
Abstracts for the Royal Australian and \\
New Zealand College of Psychiatrists 2005 \\
[38] \\
5th Joint Meeting of the European Tissue \\
Repair Society and the Wound Healing \\
Society 2009 [39] \\
Recently published abstracts [40]
\end{tabular}

DENTSPLY Posters 2012 [41]

HealthBeat 2007 [42]

Abstracts for Poster Presentations 2011 [43]

Abstracts for the International Symposium on Dental Hygiene 2013 [44]

Abou Sulaiman et al. 2010 [45]

Acharya et al. 2010 [46]

Akpinar et al. 2013 [47]

Altay et al. 2013 [20]

Armstrong et al. 2010 [48]

Arora et al. 2013 [49]

Basegmez et al. 2011 [50]

Bresolin et al. 2013 [51]

Bresolin et al. 2014 [52]

Caspersen et al. 2012 [53]

Caula et al. 2014 [54]

Caula et al. 2014 [54]

Chapple et a. 2006 [55]

Chapple et al. 2007 [56]

Chandni et al. 2015 [57]

Chaston et al. 2014 [58]

Chee et al. 2008 [59]

Chee et al. 2013 [60]

Chen et al. 2012 [61]

D'Aiuto et al. 2004 [62]

Reason for exclusion

Not addressing research

question

Not addressing research question

Not addressing research question

Not addressing research question

Not addressing research question

Not addressing research question

Not addressing research question

Not addressing research question

Not addressing research question

No PPD reduction data

Not addressing research question

No PPD reduction data

Not addressing research question

Not addressing research question

Not addressing research question

Not addressing research question

Not addressing research question

Not addressing research question

No comparison

Duplicate

Not addressing research question

Not addressing research question

Not addressing research question

Not addressing research question

Inclusion of diabetes

Missed intervention,

inclusion of diabetes

Inclusion of diabetes

Not addressing research question
Table 3 Excluded studies (Continued)

D’Aiuto et al. 2004 [63]

Not addressing research question

D'Aiuto et al. 2005 [23]

No comparison

D’Aiuto et al. 2006 [64]

Deppe et al. 2010 [65]

Dodington et al. 2015 [66]

Draper et al. 2010 [67]

Duan et al. 2009 [24]

Edwards et al. 2006 [68]

Efurd et al. 2012 [69]

Elliott-Smith et al. 2011 [70]

Engebretson et al. 2014 [71]

Fairfield et al. 2010 [72]

Fang et al. 2015 [73]

Fentoglu et al. 2010 [74]

Fine et al. 2007 [75]

Fokkema et al. 2012 [76]

Fu et al. 2015 [77]

Garcia et al. 2010 [78]

Giblin et al. 2014 [79]

Giblin et al. 2014 [79]

Gluch et al. 2007 [80]

Goldie et al. 2002 [81]

Goldie et al. 2004 [82]

Goldie et al. 2005 [83)

Griffin et al. 2012 [84]

Gurenlian et al. 2006 [85]

Gurenlian et al. 2009 [86]

Hammaker et al. 2010 [87]

Horwitz et al. 2007 [88]

Hovliaras-Delozier et al. 2008 [89]

Ide et al. 2004 [90]
Not addressing research question

Not addressing research question

No obesity data

Not addressing research question

Written in Chinese

Not addressing research question question

Not addressing research question

Not addressing research question

Not addressing research question

Inclusion of end-stage renal disease patients

Statin intake

Not addressing research question

Not addressing research question

No comparison

Not addressing research question

Inclusion of prediabetes

Duplicate

Inclusion of diabetes

Not addressing research question

Not addressing research question

Not addressing research question

Not addressing research question question

Not addressing research question

Not addressing research question

Not addressing research question

Not addressing research question

No obesity data
Not addressing research

Not addressing research 
Table 3 Excluded studies (Continued)

Ide et al. 2007 [91]

Iwamoto et al. 2003 [25]

Jahn et al. 2004 [92]

Jahn et al. 2015 [93]

Jaiswal et al. 2015 [94]

Janket et al. 2014 [95]

Janket et al. 2014 [95]

Jared et al. 2008 [96]

Jared et al. 2008 [96]

Jiang et al. 2013 [97]

Kamil et al. 2011 [98]

Kamilov et al. 1998 [99]

Kapellas et al. 2013 [100]

Kardeşler et al. 2010 [26]

Keller et al. 2015 [101]

Kiany et al. 2014 [102]

Kiany et al. 2015 [103]

Kipp et al. 2003 [104]

Kudva et al. 2010 [105]

Kumar et al. 2015 [106]

Kurti et al. 2007 [107]

Kurtis et al. 2007 [108]

Lee et al. 2014 [109]

Li et al. 2013 [110]

Ling et al. 2012 [111]

Lo et al. 2011 [112]

Malhotra et al. 2012 [113]

Mancl et al. 2013 [114]

Martinez et al. 2014 [115]

Martinez et al. 2014 [116]

Matlock et al. 2012 [117]

McDaniel et al. 2013 [118]

Meharwade et al. 2014 [119]

Merchant et al. 2014 [120]

Michalowicz et al. 2014 [121]
Not addressing research question

Application of antibiotics

Not addressing research question

Not addressing research question

No treatment (SRP)

Meta-analysis

Duplicate

Not addressing research question

Duplicate

Not addressing research question

No obesity data

Not addressing research question

No obesity data

Inclusion of diabetes

Systematic review

No obesity data

Duplicate

Not addressing research question

Inclusion of diabetes

Inclusion of diabetes

Not addressing research question

Duplicate

Not addressing research question

No obesity data

Inclusion of diabetes

Not addressing research question

No obesity data

Not addressing research question

Supplementation of $\omega-3$ eicosapetaenoic acid

One year of omega-3 supplementation

No treatment (SRP)

Not addressing research question

Local drug delivery

Inclusion of diabetes

Inclusion of diabetes

Table 3 Excluded studies (Continued)

Mizrak et al. 2006 [122]

Moeintaghavi et al. 2012 [123]

Moravec et al. 2011 [124]

Muthu et al. 2015 [125]

Nassar et al. 2012 [126]

Newton et al. 2011 [127]

Nichols et al. 2001 [128]

Nielsen et al. 2000 [129]

Novakovic et al. 2013 [130]

Novakovic et al. 2014 [131]

Oliveira et al. 2011 [132]

Olsen et al. 2006 [133]

Paquette et al. 2008 [134]

Perayil et al. 2014 [135]

Perayil et al. 2014 [136]

Phillips et al. 2013 [137]

Pradeep et al. 2007 [138]

Price et al. 2010 [139]

Qiqiang et al. 2012 [140]

Radafshar et al. 2012 [141]

Radnai et al. 2009 [142]

Raghavendra et al. 2012 [143]

Ramirez et al. 2011 [144]

Rasch et al. 1995 [145]

Sadatmansouri et al. 2006 [146]

Saffi et al. 2013 [147]

Saffi et al. 2013 [147]

Saffi et al. 2014 [148]

Shruti et al. 2010 [27]

Sembene et al. 2000 [149]

Sengupta et al. 1990 [150]
Not addressing research question

Inclusion of diabetes

No treatment (SRP)

No obesity data

Inclusion of diabetes

Not addressing research question

Not addressing research question

Not addressing research question

Not addressing research question

Not addressing research question

Not addressing research question

Not addressing research question

Not addressing research question

No obesity data

Duplicate

Not addressing research question

Not addressing research question

Not addressing research question

No obesity data

Not addressing research question

Not addressing research question

Not addressing research question

No obesity data

Not addressing research question

Not addressing research question

Inclusion of patients with coronary disease

Duplicate

Inclusion of patients with coronary artery disease

No obesity data

Not addressing research question

Not addressing research question 
Table 3 Excluded studies (Continued)

\begin{tabular}{|c|c|}
\hline Sgolastra et al. 2013 [36] & Meta-Analysis \\
\hline Shimada et al. 2010 [151] & No comparison \\
\hline Shimoe et al. 2011 [152] & Case report \\
\hline Singh et al. 2014 [153] & $\begin{array}{l}\text { Not addressing research } \\
\text { question }\end{array}$ \\
\hline Siqueira et al. 2013 [154] & $\begin{array}{l}\text { Not addressing research } \\
\text { question }\end{array}$ \\
\hline Stewart et al. 2001 [155] & Inclusion of diabetes \\
\hline Talbert et al. 2006 [156] & Inclusion of diabetes \\
\hline Tamaki et al. 2009 [157] & No obesity data \\
\hline Tamaki et al. 2011 [158] & No obesity data \\
\hline Tawfig et al. 2015 [159] & No comparison \\
\hline Teles et al. 2012 [160] & No comparison \\
\hline Toker et al. 2012 [161] & $\begin{array}{l}\text { Not addressing research } \\
\text { question }\end{array}$ \\
\hline Tuter et al. 2007 [162] & $\begin{array}{l}\text { Inclusion of patients with } \\
\text { systemic disease }\end{array}$ \\
\hline Vardar et al. 2003 [163] & $\begin{array}{l}\text { Not addressing research } \\
\text { question }\end{array}$ \\
\hline Van Dyke et al. 2015 [164] & $\begin{array}{l}\text { Not addressing research } \\
\text { question }\end{array}$ \\
\hline Vyas et al. 2000 [165] & $\begin{array}{l}\text { Not addressing research } \\
\text { question }\end{array}$ \\
\hline Wahid et al. 2013 [166] & $\begin{array}{l}\text { Not addressing research } \\
\text { question }\end{array}$ \\
\hline Wang et al. 2013 [167] & $\begin{array}{l}\text { Inclusion of aggressive } \\
\text { periodontitis }\end{array}$ \\
\hline Wehmeyer et al. 2013 [168] & $\begin{array}{l}\text { Not addressing research } \\
\text { question }\end{array}$ \\
\hline Wei et al. 2010 [169] & No obesity data \\
\hline Williams et al. 2007 [148] & No obesity data \\
\hline Williams et al. 2009 [170] & $\begin{array}{l}\text { Not addressing research } \\
\text { question }\end{array}$ \\
\hline Williams et al. 2009 (171] & $\begin{array}{l}\text { Not addressing research } \\
\text { question }\end{array}$ \\
\hline Wood et al. 2006 [172) & $\begin{array}{l}\text { Not addressing research } \\
\text { question }\end{array}$ \\
\hline Wu et al. 2015 [173) & $\begin{array}{l}\text { Not addressing research } \\
\text { question }\end{array}$ \\
\hline Zare et al. 2014 [174) & $\begin{array}{l}\text { Not addressing research } \\
\text { question }\end{array}$ \\
\hline Zhou et al. 2013 [175] & $\begin{array}{l}\text { Inclusion of patients with } \\
\text { systemic disease }\end{array}$ \\
\hline Zuza et al. 2011 [176) & No PPD reduction data \\
\hline
\end{tabular}

criteria (Table 3). Therefore, eight publications $[8,28-$ 34] from the electronic and hand search fulfilled the criteria. However, it was not possible to compare the raw data so that we had to reduce our analysis to a qualitative analysis. This process is summarized in a flow-chart (Fig. 1).
Summary of studies: characteristics (PICO)

Total number of patients, demographic data, origin of study, outcome measurements 2, 3, 6 and 12 months after therapy and the impact of obesity on the treatmentoutcome are summarized in Table 4.

Definition of chronic periodontitis, periodontal assessment and the amount of smokers included are depicted in Table 5.

The definition of obesity and systemic examination were summarized in Table 6. Non-surgical periodontal treatment, treatment time, periodontal maintenance and limitations are shown in Table 7.

\section{Population}

Of the eight finally analyzed studies, clinical trials comprised 516 participants. One study [8] enrolled only women. The prevalence of smoking among male patients affected with periodontitis was so high that Al-Zahrani and co-workers [8] were unable to correct for smoking in the male population. Accordingly, only women were included in their study. Overall the studies comprised between 26 up to 260 subjects (Table 4).

The inclusion criteria "age" was defined in all but one study [30]. Three investigations [28, 33, 34] defined the age $\geq 18$ years. The other studies defined a minimum age of 30 years [31, 32] or 35 years [8]. Duzagac et al. defined an age range from 25 to 55 years (Table 4).

Suvan et al. [34] included smokers into their study. Lakkis et al. [33] and Eldin et al.(30] did not mention the smoking status of the patients. All other studies $[8$, 28, 29, 31, 32] excluded smokers (Table 5).

Patients with diabetes, another important modifier of periodontal health or disease [35] were excluded in seven studies [8, 28-32, 34]. Lakkis et al. [33] did not report the presence or absence of diabetes.

\section{Intervention/Comparison}

Each paper described the periodontal intervention as a non-surgical therapy. All studies [8, 28-34] applied scaling and root planing. Ultrasonic instruments and/or hand instruments were used in all studies (Table 7).

Two papers $(8,34]$ reassessed the PPD 2 months after therapy. Two studies $[29,30]$ reassessed their patients after 3 months. Another two papers [28, 31] reevaluated the patients after 3 and 6 months and one study [32] reassessed the PPD three, six and 12 months after therapy. Only Lakkis et al. [33] measured the periodontal pocket depths already 4 to 6 weeks after nonsurgical therapy (Table 3). Oral hygiene was instructed additionally to the non-surgical periodontal therapy in all studies (Table 7).

Due to the heterogeneity of the study designs with respect to outcome measures and treatment protocol, as well as variation in study population, sample size, and/or 
Table 4 Probing pocket depth (PPD [mm]) as primary outcome variable

\begin{tabular}{|c|c|c|c|c|c|c|c|}
\hline Author \& year & $\begin{array}{l}\text { Total number of patients } \\
\text { Age (mean } \pm \text { SD) } \\
\text { Country }\end{array}$ & $\begin{array}{l}\text { Outcome after } 2 \text { months } \\
\text { mean }( \pm \text { SD) at baseline - } \\
\text { mean }( \pm \text { SD) at reassessment }\end{array}$ & $\begin{array}{l}\text { Outcome after } 3 \\
\text { months } \\
\text { mean }( \pm S D) \\
\text { at baseline }- \\
\text { mean }( \pm \text { SD) } \\
\text { at reassessment }\end{array}$ & $\begin{array}{l}\text { Outcome after } 6 \text { months } \\
\text { mean }( \pm \text { SD)at baseline - } \\
\text { mean }( \pm \text { SD) at } \\
\text { reassessment }\end{array}$ & $\begin{array}{l}\text { Outcome after } \\
12 \text { months } \\
\text { mean }( \pm \text { SD)at } \\
\text { baseline - mean } \\
( \pm \text { SD) at } \\
\text { reassessment }\end{array}$ & \multicolumn{2}{|c|}{$\begin{array}{l}\text { Impact of obesity on the treatment - } \\
\text { outcome } \\
\text { mean - reduction of } \\
\text { PPD ( } \pm \text { SD) }\end{array}$} \\
\hline $\begin{array}{l}\text { Al - Zahrani } \\
\text { et al. } 2012[8]\end{array}$ & $\begin{array}{l}\text { Total number of patients: } \\
40 \text { (only women) } \\
\text { Normal-weight: } 20 \\
\text { Obese: } 20 \\
\text { Age: } \\
\text { Normal-weight: } 43.4( \pm 7.8) \\
\text { Obese: } 44 \text { ( } \pm 8.4) \\
\text { Country: } \\
\text { Saudi Arabia }\end{array}$ & $\begin{array}{l}\text { Total sample: } \\
2.65( \pm 0.47)-2.44( \pm 0.41) \\
p \text {-value }=0.001\end{array}$ & n.r. & n.r. & n.r. & \multicolumn{2}{|c|}{$\begin{array}{l}\text { Normal-weight: } \\
0.19( \pm 0.27) \\
\text { Obese: } \\
0.23( \pm 0.33) \\
p \text {-value }=0.663 \text { (n. sig.) }\end{array}$} \\
\hline $\begin{array}{l}\text { Bouaziz et al. } \\
2015 \text { [28] }\end{array}$ & $\begin{array}{l}\text { Total number of patients: } \\
36 \\
\text { Normal-weight: } 18 \\
\text { Obese: } 18 \\
\text { Age: } \\
\text { Range from } 22 \text { to } 78 \\
\text { Normal-weight: } 51.00( \pm 13.1) \\
\text { Obese: } 51.3( \pm 16.7) \\
\text { p-value: } n \text {. sig. } \\
\text { Country: } \\
\text { France }\end{array}$ & n.r. & $\begin{array}{l}\text { Normal-weight: } 3.33 \\
( \pm 0.92)-2.71( \pm 0.40) \\
p \text {-value: } n . \text { sig. } \\
\text { Obese: } 3.22( \pm 0.76)- \\
2.78( \pm 0.55) \\
p \text {-value: n. sig. }\end{array}$ & $\begin{array}{l}\text { Normal-weight: } 3.33 \\
( \pm 0.92) 2.45( \pm 0.53) \\
p \text {-value }<0.05 \\
\text { Obese: } 3.22( \pm 0.76)- \\
2.43( \pm 0.49) \\
p \text {-value }<0.05\end{array}$ & n.r. & \multicolumn{2}{|c|}{$\begin{array}{l}\text { Summary of Multivariable Analysis } \\
\text { Results (Coefficient, } 95 \% \mathrm{Cl}) \text { : } \\
\Delta \% \mathrm{PD}>5 \mathrm{~mm} \text { after } 3 \text { months to obese } \\
(\text { Model } 2): \\
p \text {-value }=0.02 \\
\Delta \% \mathrm{PD}>5 \mathrm{~mm} \text { after } 6 \text { months to obese } \\
\text { (Model 2): } \\
p \text {-value }=0.005 \\
\text { Nb } \Delta \mathrm{PD}>2 \mathrm{~mm} \text { after } 3 \text { months to Obese } \\
\text { (Model 2); } \\
p \text {-value }=0.02 \\
\text { Nb } \triangle \mathrm{PD}>2 \mathrm{~mm} \text { after } 6 \text { months to Obese } \\
(\text { Model } 2) ; \\
p \text {-value }=0.06 \text { (n.sig.) }\end{array}$} \\
\hline $\begin{array}{l}\text { Duzagac et al. } \\
2015 \text { [29] }\end{array}$ & $\begin{array}{l}\text { Total number of patients: } \\
45 \\
\text { Normal weight patients with } \\
\text { periodontitis (CPNW): } 15 \\
\text { Obese patients with periodontitis } \\
\text { (CPO): } \\
15 \\
\text { Normal weight periodontally } \\
\text { healthy control subjects (PHNW): } 15 \\
\text { Age: } \\
\text { Range from } 28 \text { to } 52 \\
\text { CPNW: } 41.06 \text { ( } \pm 7.10) \\
\text { CPO: } 40.66 \text { ( } \pm 7.98) \\
\text { PHNW: } 39.66 \text { ( } \pm 6.53) \\
\text { p-value: } n \text {. sig. } \\
\text { Country: } \\
\text { Turkey }\end{array}$ & n.r. & $\begin{array}{l}\text { CPNW: } 3.19( \pm 0.38)- \\
2.51-( \pm 0.06) \\
p \text {-value }=0.001 \\
\text { CPO: } 4.10( \pm 0.50)- \\
2.55( \pm 0.45) \\
p \text {-value }=0.001\end{array}$ & n.r. & n.r. & $\begin{array}{l}\text { Number of sites } \\
\text { with PD < } 4 \mathrm{~mm} \text { : } \\
\text { Baseline: } \\
\text { CPO: } 1706 \\
\text { CPNW: } 1766 \\
3 \text { months: } \\
\text { CPO: } 2214 \\
\text { CPNW: } 2206 \\
\% \text { of sites with PD } \\
\text { < } 4 \text { mm: } \\
\text { Baseline: } \\
\text { CPO: } 75.4 \\
\text { CPNW: } 77.9 \\
3 \text { months: } \\
\text { CPO: } 97.9 \\
\text { CPNW: } 97.3 \\
\text { Similar clinical } \\
\text { healing; } \\
\text { (n. sig.) }\end{array}$ & $\begin{array}{l}\text { Number of sites with } \\
\text { PD } \geq 4 \text { mm: } \\
\text { Baseline: } \\
\text { CPO: } 556 \\
\text { CPNW: } 502 \\
3 \text { months: } \\
\text { CPO: } 48 \\
\text { CPNW: } 32 \\
\% \text { of sites with PD } \geq \\
4 \text { mm: } \\
\text { Baseline: } \\
\text { CPO: } 48 \\
\text { CPNW: } 22.1 \\
3 \text { months: } \\
\text { CPO: } 2.1 \\
\text { CPNW: } 2.7 \\
\text { Similar clinical } \\
\text { healing; } \\
\text { (n. sig.) }\end{array}$ \\
\hline
\end{tabular}

. $22(+0.76)-$

$2.78( \pm 0.55)$

p-value $<0.05$

Obese: $3.22( \pm 0.76)-$

$2.43( \pm 0.49)$

Range from 22 to 78

Normal-weight: $51.00( \pm 13.1)$

Country:

Total number of patients:

Obese patients with periodontitis

15

Age:

from 28 to 52

PHNW: $39.66( \pm 6.53)$

Country:

(n. sig.)

(n. sig.) 
Table 4 Probing pocket depth (PPD [mm]) as primary outcome variable (Continued)

\begin{tabular}{|c|c|c|c|c|c|c|c|c|}
\hline $\begin{array}{l}\text { Eldin et al. } \\
2013 \text { [30] }\end{array}$ & $\begin{array}{l}\text { Total number of patients: } \\
26 \\
\text { Overweight: } 12 \\
\text { Obese: } 14 \\
\text { Age: } \\
\text { Range from } 35 \text { to } 48 \\
\text { Country: } \\
\text { Egypt }\end{array}$ & n.r. & $\begin{array}{l}\text { Overweight: } 0.6( \pm 1.29) \\
-0.75 \text { ( } \pm 2.12) \\
\text { p-value: } n . r . \\
\text { Obese: } 3.15( \pm 2.43)- \\
1.59( \pm 2.34) \\
p \text {-value: } n . r .\end{array}$ & n.r. & n.r. & $\begin{array}{l}\text { Overweight: } \\
0.784 \\
\text { Obese: } \\
0.209 \\
p \text {-value }=0.326(\mathrm{n} \text {. }\end{array}$ & & \\
\hline $\begin{array}{l}\text { Gonçalves } \\
\text { et al. } 2015 \text { [31] }\end{array}$ & $\begin{array}{l}\text { Total number of patients: } \\
39 \\
\text { Without obesity: } 21 \\
\text { With obesity: } 18 \\
\text { Age: } \\
\text { Without obesity: } 48.4( \pm 9.5) \\
\text { With obesity: } 48.8( \pm 5.9) \\
\text { p-value= } 0.85 \text { (n. sig.) } \\
\text { Country: } \\
\text { Brazil }\end{array}$ & n.r. & $\begin{array}{l}\text { Without obesity: } 3.4 \\
( \pm 0.6)-3.0( \pm 0.5) \\
p \text {-value: sig. } \\
\text { With obesity: } 3.6( \pm 0.6) \\
-3.3 \text { ( } \pm 0.6) \\
p \text {-value: sig. }\end{array}$ & $\begin{array}{l}\text { Without obesity: } \\
3.4 \text { ( } \pm 0.6)-2.7( \pm 0.6) \\
\text { p-value: sig. } \\
\text { With obesity: } \\
3.6 \text { ( } \pm 0.6) \text { - } 3.0( \pm 0.5) \\
p \text {-value: sig. }\end{array}$ & n.r. & $\begin{array}{l}\text { Without obesity } \\
\text { Full-mouth sites: } \\
0-3 \text { months: } 0.44 \\
( \pm 0.06) \\
0-6 \text { months: } 0.73 \\
( \pm 0.07) \\
\text { Initially moderate } \\
\text { sites } \\
\text { (PD 4-6mm): } \\
0-3 \text { months: } 0.87 \\
( \pm 0.12) \\
0-6 \text { months: } 1.38 \\
( \pm 0.14) \\
\text { Initially deep sites } \\
\text { (PD } \geq 7 m m): \\
0-3 \text { months: } 1.86 \\
( \pm 0.25) \\
0-6 \text { months: } 3.00 \\
( \pm 0.23)\end{array}$ & $\begin{array}{l}\text { With obesity } \\
\text { Full-mouth } \\
\text { sites: } \\
0-3 \text { months: } \\
0.30 \text { ( } \pm 0.06) \\
0-6 \text { months: } \\
0.54 \text { ( } \pm 0.07) \\
\text { Initially } \\
\text { moderate } \\
\text { sites } \\
\text { (PD 4-6mm): } \\
0-3 \text { months: } \\
0.70 \text { ( } \pm 0.13) \\
0-6 \text { months: } \\
1.10( \pm 0.15) \\
\text { Initially deep } \\
\text { sites } \\
\text { (PD } \geq 7 \mathrm{~mm}): \\
0-3 \text { months: } \\
1.22 \text { ( } \pm 0.26) \\
0-6 \text { months: } \\
2.30 \text { ( } \pm 0.24)\end{array}$ & $\begin{array}{c}p \text {-value } \\
(<0.05) \\
0.08 \\
0.04 \\
0.31 \\
0.16 \\
0.07 \\
0.04 \\
\end{array}$ \\
\hline $\begin{array}{l}\text { Gonçalves } \\
\text { et al. } 2015 \\
\text { [32] }\end{array}$ & $\begin{array}{l}\text { Total number of patients: } \\
40 \\
\text { Non-obese: } 20 \\
\text { Obese: } 20 \\
\text { Age: } \\
\text { Non-obese: } 48.5( \pm 9.3) \\
\text { Obese: } 50.0( \pm 4.5) \\
\text { p-value }=0.52 \text { (n. sig.) } \\
\text { Country: } \\
\text { Brazil }\end{array}$ & n.r. & $\begin{array}{l}\text { Non-obese: } 3.4( \pm 0.6)- \\
2.9 \text { ( } \pm 0.6) \\
\text { p-value: sig. } \\
\text { Obese: } 3.6( \pm 0.6)-3.3 \\
( \pm 0.5) \\
p \text {-value: sig. }\end{array}$ & $\begin{array}{l}\text { Non-obese: } 3.4( \pm 0.6)- \\
2.7 \text { ( } \pm 0.6) \\
\text { p-value: sig. } \\
\text { Obese: } 3.6( \pm 0.6)-3.0 \\
( \pm 0.5) \\
p \text {-value: sig. }\end{array}$ & $\begin{array}{l}\text { Non-obese: } 3.4 \\
( \pm 0.6)-2.6( \pm 0.6) \\
\text { p-value: sig. } \\
\text { Obese: } 3.6 \\
( \pm 0.6)-2.9( \pm 0.4) \\
p \text {-value: sig. }\end{array}$ & \multicolumn{3}{|c|}{$\begin{array}{l}\text { Mean PPD values }( \pm \text { SD): } \\
\text { After } 3 \text { months: } \\
\text { Non-obese: } 2.9( \pm 0.6) \\
\text { Obese: } 3.3( \pm 0.5) \\
p=0.25 \text { (n. sig.) } \\
\text { After } 6 \text { months: } \\
\text { Non-obese: } 2.7( \pm 0.6) \\
\text { Obese: } 3.0( \pm 0.5) \\
p=0.04 \\
\text { After } 12 \text { months: } \\
\text { Non-obese: } 2.6( \pm 0.6) \\
\text { Obese: } 2.9( \pm 0.4) \\
p=0.03\end{array}$} \\
\hline
\end{tabular}


Table 4 Probing pocket depth (PPD [mm]) as primary outcome variable (Continued)

\begin{tabular}{|c|c|c|c|c|c|c|}
\hline $\begin{array}{l}\text { Lakkis et al. } \\
2011 \text { [33] }\end{array}$ & $\begin{array}{l}\text { Total number of patients: } \\
30 \\
\text { Surgery: } 15 \\
\text { Control: } 15 \\
\text { Age: } \\
\text { Surgery: } 45.6( \pm 11.1) \\
\text { Control: } 48.5( \pm 12.0) \\
\text { p-value }=0.778 \text { (n. sig.) } \\
\text { Country: } \\
\text { USA (Cleveland, Ohio) }\end{array}$ & $\begin{array}{l}\text { 4-6 weeks after therapy! } \\
\text { Surgery: } \\
2.6( \pm 0.6)-2.2( \pm 0.5) \\
p \text {-value }<0.001 \\
\text { Control: } \\
3.1( \pm 0.8)-2.8( \pm 0.7) \\
p \text {-value }<0.001\end{array}$ & n.r. & n.r. & n.r. & $\begin{array}{l}\text { Surgery: } \\
0.45 \\
\text { Control: } \\
0.28 \\
p \text {-value }=0.007\end{array}$ \\
\hline $\begin{array}{l}\text { Suvan et al. } \\
2014 \text { [34] }\end{array}$ & $\begin{array}{l}\text { Total number of patients: } \\
260 \\
\text { Normal: } 112 \\
\text { Overweight: } 93 \\
\text { Obese: } 55 \\
\text { Age: } \\
\text { Normal: } 46.30( \pm 8.38) \\
\text { Overweight: } 47.27( \pm 8.93) \\
\text { Obese: } 46.55( \pm 6.87) \\
\text { p-value }=0.701 \text { (n. sig.) } \\
\text { Country: } \\
\text { Great Britain (London) }\end{array}$ & $\begin{array}{l}\text { Summary of univariable } \\
\text { GEE Analysis Results } \\
\text { (Coefficient, } 95 \% \mathrm{Cl} \text { ): } \\
\text { BMl }\left[\mathrm{kg} / \mathrm{m}^{2}\right] \text { to PPD } \\
\text { at } 2 \text { months } \\
p \text {-value }=0.055 \\
\text { Overweight to PPD } \\
\text { at } 2 \text { months } \\
p \text {-value }=0.372 \text { (n. sig.) } \\
\text { Obese to PPD at } 2 \text { months } \\
p \text {-value }=0.064 \text { (n. sig.) } \\
\text { Summary of Multivariable } \\
\text { GEE Analysis Results } \\
\text { (Coefficient, } 95 \% \text { Cl): } \\
\text { BMl [kg/m²] to PPD } \\
\text { at } 2 \text { months } \\
p \text {-value }=0.013 \\
\text { Overweight to PPD } \\
\text { at } 2 \text { months } \\
p \text {-value }=0.63 \text { (n. sig.) } \\
\text { Obese to PPD at } 2 \text { months } \\
p \text {-value }=0.031\end{array}$ & n.r. & n.r. & n.r. & $\begin{array}{l}\text { BMI and obesity were sig. associated with } \\
\text { mean PPD after } 2 \text { months. }(p=0.055) \\
\text { Overweight was not sig. associated with } \\
\text { PPD after } 2 \text { months }(p=0.372) \\
\text { BMI had a stat. sig. linear relationship with } \\
\text { mean PPD after } 2 \text { months }(p=0.013) \text {. } \\
\text { Overweight compared with normal BMI } \\
\text { status did not demonstrate a stat. sig. } \\
\text { association with mean PPD after } 2 \\
\text { months ( } p=0.63) \text {. Obesity compared } \\
\text { with normal BMl status remained a stat. } \\
\text { sig. predictor of mean PPD ( } p=0.031) \text { at } \\
2 \text { months in the same multivariable } \\
\text { model. }\end{array}$ \\
\hline
\end{tabular}

depth, PHNW normal weight periodontally healthy control subjects, PPD periodontal pocket depth, $p$ probability, SD standard deviation, (n.) sig. (not) significant, stat. statistically, WHO world health organisation 
Table 5 Definition of periodontitis, periodontal assessment and definition of smoking

\begin{tabular}{|c|c|c|c|}
\hline Author \& year & Definition of chronic periodontitis & Periodontal assessment & Smokers \\
\hline $\begin{array}{l}\text { Al - Zahrani } \\
\text { et al. } 2012[8]\end{array}$ & $\begin{array}{l}\geq 20 \text { teeth, } \\
\text { CAL } \geq 3 \mathrm{~mm} \text { at } \geq 30 \text { of the sites } \\
\text { (generalized moderate/severe chronic periodontits) }\end{array}$ & $\begin{array}{l}\text { Full-mouth periodontal examination on all teeth } \\
\text { excluding 3rd molars and partially erupted teeth }\end{array}$ & Smokers excluded \\
\hline $\begin{array}{l}\text { Bouaziz et al. } \\
2015 \text { [28] }\end{array}$ & $\begin{array}{l}\geq 12 \text { teeth, } \\
\geq 30 \% \text { of sites with } C A L \geq 3 \mathrm{~mm} \\
\text { (moderate-to-severe generalized chronic } \\
\text { periodontitis) }\end{array}$ & $\begin{array}{l}\text { Full-mouth clinical measurements included the } \\
\text { following: } \\
\text { 1) PI, } \\
\text { 2) BOP, } \\
\text { 3) PD (mm), } \\
\text { 4) CAL (mm), } \\
\text { Pl, BOP, PD, and CAL measurements were performed } \\
\text { using a manual periodontal probe at } 6 \text { sites per tooth, } \\
\text { PD was defined as the distance from the free gingival } \\
\text { margin to the bottom of the pocket/sulcus, and CAL } \\
\text { was defined as the distance from the cemento-enamel } \\
\text { junction to the bottom of the pocket/sulcus }\end{array}$ & $\begin{array}{l}\text { Non-smoker or } \\
\text { former smoker } \\
\text { since } \geq 5 \text { years }\end{array}$ \\
\hline $\begin{array}{l}\text { Duzagac et al. } \\
2015 \text { [29] }\end{array}$ & $\begin{array}{l}\geq 20 \text { teeth, } \\
\mathrm{PD} \geq 4 \mathrm{~mm} \text { in } \geq 30 \% \text { of periodontal sites, } \\
\mathrm{BOP} \text { in } \geq 50 \% \text { of periodontal sites, } \\
\text { interproximal CAL }>2 \mathrm{~mm} \text { in } \geq 20 \% \text { of periodontal } \\
\text { sites, } \\
\text { radiographic evidence of bone loss, as described } \\
\text { by Armitage [177] }\end{array}$ & $\begin{array}{l}\text { A Williams-type periodontal probe (UNC15, Hu-Friedy, } \\
\text { Chicago, IL, USA) was used for the measurements of } \\
\text { periodontal parameters, including PI (Silness-Löe) } \\
\text { [178], GI (Löe-Silness) [179], BOP, PD and CAL, } \\
\text { all clinical periodontal measurements were performed } \\
\text { at } 6 \text { sites per tooth (mesiobuccal, mid-buccal, distobuccal, } \\
\text { mesiolingual, mid-lingual and distolingual), } \\
\text { excluding third molars }\end{array}$ & Smokers excluded \\
\hline $\begin{array}{l}\text { Eldin et al. } \\
2013 \text { [30] }\end{array}$ & $\begin{array}{l}\text { n.r. } \\
\text { (chronic periodontitis) }\end{array}$ & $\begin{array}{l}\text { Full-mouth, } \\
4 \text { sites per tooth (mesio-buccal, mid-buccal, disto-buccal, } \\
\text { mid-lingual) around each tooth }\end{array}$ & n.r. \\
\hline $\begin{array}{l}\text { Gonçalves } \\
\text { et al. } 2015 \\
\text { [31] }\end{array}$ & $\begin{array}{l}\geq 15 \text { teeth excluding 3rd molars and teeth with } \\
\text { advanced decay indicated for exodontia, } \\
>30 \% \text { of the sites with concomitant PD and } \\
\text { CAL } \geq 4 \mathrm{~mm} \text { and a minimum of } 6 \text { teeth distributed } \\
\text { in the different quadrants presenting at least one site } \\
\text { with PD and CAL } \geq 5 \mathrm{~mm} \text { and BOP at baseline } \\
\text { (generalized chronic periodontitis) }\end{array}$ & $\begin{array}{l}6 \text { sites per tooth on all teeth excluding } 3 r d \text { molars } \\
\text { (distance between the gingival margin and the bottom } \\
\text { of the sulcus/pocket [mm]) }\end{array}$ & Smokers excluded \\
\hline $\begin{array}{l}\text { Gonçalves } \\
\text { et al. } 2015 \\
\text { [32] }\end{array}$ & $\begin{array}{l}\geq 15 \text { teeth excluding } 3 \text { rd molars and teeth with } \\
\text { advanced decay indicated for exodontia, } \\
>30 \% \text { of the sites with concomitant PD and } \\
\text { CAL } \geq 4 \mathrm{~mm} \text { and a minimum of } 6 \text { teeth distributed } \\
\text { in the different quadrants presenting at least one site } \\
\text { with PD and CAL } \geq 5 \mathrm{~mm} \text { and BOP at baseline } \\
\text { (generalized chronic periodontitis) }\end{array}$ & $\begin{array}{l}6 \text { sites per tooth (mesio-buccal, mid-buccal, disto-buccal, } \\
\text { mesio-lingual, mid-lingual, disto-lingual) excluding third } \\
\text { molars, with a manual periodontal probe (UNC15, } \\
\text { Hu-Friedy, Chicago, IL, USA) }\end{array}$ & $\begin{array}{l}\text { Current smokers } \\
\text { excluded, smoking } \\
\text { within the past } \\
10 \text { years excluded }\end{array}$ \\
\hline $\begin{array}{l}\text { Lakkis et al. } \\
2011 \text { [33] }\end{array}$ & $\begin{array}{l}\geq 20 \text { teeth, } \\
\text { mean } C A L \geq 2 \mathrm{~mm} \\
\text { (chronic periodontitis) }\end{array}$ & 6 sites per tooth & n.r. \\
\hline $\begin{array}{l}\text { Suvan et al. } \\
2014 \text { [34] }\end{array}$ & $\begin{array}{l}\mathrm{PD}>5 \mathrm{~mm} \text { and marginal alveolar bone } \\
\text { loss }>30 \% \text { with }>50 \% \text { of the teeth affected } \\
\text { (generalized severe periodontitis) }\end{array}$ & 6 sites per tooth on all teeth present & $\begin{array}{l}\text { Normal-group }= \\
47 \% \text { smokers } \\
\text { Overweight-group = } \\
25 \% \text { smokers } \\
\text { Obese-group }= \\
15 \% \text { smokers } \\
p-\text { value }=0.045 \\
\text { (sig. on } p \leq 0.05 \text { ) }\end{array}$ \\
\hline
\end{tabular}

BoP bleeding on probing, CAL clinical attachment level, $G$ g gingival index, $m m$ millimeter, n.r. not reported, $p$ probability, $P D$ probing depth, $P I$ plaque index, sig. significant

statistical methods, a statistical synthesis of the results of the included studies was not possible. So the authors decided to analyze the papers on a qualitative way [22]. A meta-analysis was not possible.

\section{Outcome}

Generally obese patients were found to have deeper periodontal pockets at baseline in all studies.
Three $[8,29,30]$ of the eight papers [8, 28-34] reported no major negative impact of obesity on response to periodontal therapy in terms of PPD reduction ( $\mathrm{mm}$ ). Al-Zahrani et al. [8] assessed the reduction of PPD (mm) comparing obese with normal-weighted women. There was no statistically significant effect of obesity on treatment outcome. Duzagac et al. [29] assessed the clinical response to non-surgical periodontal treatment, 
Table 6 Definition of obesity and systemic examination

\begin{tabular}{|c|c|c|}
\hline Author \& year & Definition of obesity & Systemic examination \\
\hline $\begin{array}{l}\text { Al - Zahrani } \\
\text { et al. } 2012 \text { [8] }\end{array}$ & $\begin{array}{l}\text { Normal-weight: BMl: } 18.5 \text { to }<25 \mathrm{~kg} / \mathrm{m}^{2} \\
\text { Obese: } \mathrm{BMI} \geq 30 \mathrm{~kg} / \mathrm{m}^{2}\end{array}$ & $\begin{array}{l}\text { Weight in kilograms and height in meters were measured; } \\
\text { BMI: (weight/height }{ }^{2}\left[\mathrm{~kg} / \mathrm{m}^{2}\right] \text { ) }\end{array}$ \\
\hline $\begin{array}{l}\text { Bouaziz et al. } \\
2015 \text { [28] }\end{array}$ & $\begin{array}{l}\text { Normal-weight: } \mathrm{BMI} \geq 18.5 \mathrm{~kg} / \mathrm{m}^{2} \text { and } \leq 25 \mathrm{~kg} / \mathrm{m}^{2} \\
\text { Obese: } \mathrm{BMI} \geq 30 \mathrm{~kg} / \mathrm{m}^{2}\end{array}$ & $\begin{array}{l}\text { Weight }(\mathrm{kg}) \text {, height }(\mathrm{m}) \text {, waist/hip circumferences }(\mathrm{cm}) \\
\text { weight was obtained using a calibrated scale, } \\
\text { height was measured using a measuring board, } \\
\left.\text { BMl: (weight/height }{ }^{2}\left[\mathrm{~kg} / \mathrm{m}^{2}\right]\right) \text {, } \\
\text { waist measurements were taken at the level of the umbilicus }(\mathrm{cm}) \\
\text { hip measurements were taken at the greatest circumference with a } \\
\text { measuring tape, } \\
\text { WHR: ratio of waist to hip circumference }\end{array}$ \\
\hline
\end{tabular}

\section{Duzagac et al. Normal-weight (CPNW): n.r. \\ 2015 [29] Obese (CPO): $\mathrm{BMI} \geq 30 \%$ and WHR $>0.95$ for males and 0,80 for females}

\author{
Eldin et al. Overweight: BMl: $25-29.9 \mathrm{~kg} / \mathrm{m}^{2}$ \\ 2013 [30] Obese: $\mathrm{BMI} \geq 30 \mathrm{~kg} / \mathrm{m}^{2}$ \\ According to the WHO (3) \\ Gonçalves et al. Without obesity: BMl $20-29.9 \mathrm{~kg} / \mathrm{m}^{2}$ and \\ 2015 [31] WHR $<0.85$ for females and $<0.9$ for males \\ With obesity: $\mathrm{BMI} \geq 30 \mathrm{~kg} / \mathrm{m}^{2}$ and $<40 \mathrm{~kg} / \mathrm{m}^{2}$ and \\ $W H R \geq 0.85$ for females and WHR $\geq 0.9$ for males
}

Non-obese: BMI: $20-24.9 \mathrm{~kg} / \mathrm{m} 2$ and WHR below that determined for obesity (i.e. WHR $<0.85$ for women and WHR $<0.90$ for men) (World Health Organization 2008) Obese: $\mathrm{BMI} \geq 30$ and $<40 \mathrm{~kg} / \mathrm{m} 2$ and concomitant WHR

$\geq 0.85$ for women and $W H R \geq 0.90$ for men.

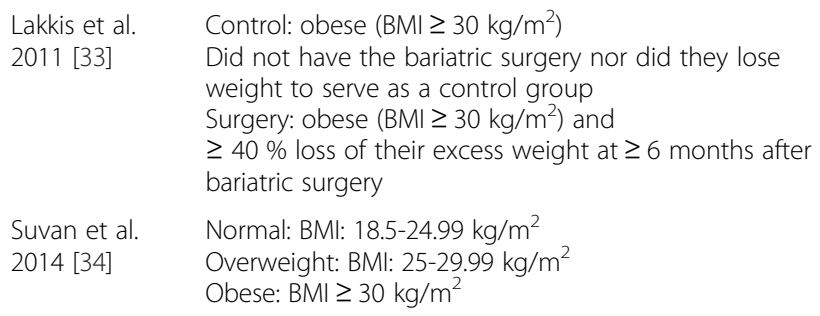

One trained examiner performed all anthropometric measurements: weight $(\mathrm{kg})$, height $(\mathrm{m})$, waist $(\mathrm{cm})$, hip $(\mathrm{cm})$, BMI: (weight/height ${ }^{2}\left[\mathrm{~kg} / \mathrm{m}^{2}\right]$ ),

WHR: ratio of waist to hip circumference, anthropometric measurements were repeated before the final follow-up visit to assess

whether there was any change in BMI or WHR

n.r.

BMI: (weight $/$ height ${ }^{2}\left[\mathrm{~kg} / \mathrm{m}^{2}\right]$ )

WHR: ratio of waist to hip circumference

One trained examiner performed

all anthropometric measurements:

weight $(\mathrm{kg})$, height $(\mathrm{m})$, waist $(\mathrm{cm})$ and

hip circumferences $(\mathrm{cm})$

BMl: (weight/height $\left.{ }^{2}\left[\mathrm{~kg} / \mathrm{m}^{2}\right]\right)$,

WHR: ratio of waist to hip circumference,

anthropometric measurements were reassessed at all follow-up visits to verify that the patients did not change their obese or non-obese status during the course of the study

BMI: (weight $/$ height ${ }^{2}\left[\mathrm{~kg} / \mathrm{m}^{2}\right]$ )

Percentage of weight loss after BS

BMI: (weight $/$ height ${ }^{2}\left[\mathrm{~kg} / \mathrm{m}^{2}\right]$ )

Based upon height and weight of the individual measured with a wall-mounted height measure and mechanical scales

$B F$ body fat, $B M I$ body mass index, $B S$ bariatric surgery, $\mathrm{Cm}$ centimeter, $C P N W$ normal weight patients with periodontitis, $C P O$ obese patients with periodontitis, $H D L$ high-density lipoprotein, $k g$ kilogram, $m$ meter, $n$. $r$. not reported, WC waist circumference, WHO world health organization, WHR waist-hip-ratio

according to the severity of periodontitis based on probing depth $<4 \mathrm{~mm}$ vs. $\geq 4 \mathrm{~mm}$. Patients with and without obesity showed similar clinical healing in terms of percentage and number of sites with probing depth $<4 \mathrm{~mm}$ and $\geq 4 \mathrm{~mm}$. So they failed to show an effect of obesity on the treatment outcome dependent on the severity of the disease. Eldin et al. [30] also found no effect when comparing an overweight group with an obese group. The difference between the groups in reduction of PPD was not significant (Table 4).

Five $[28,31-34]$ of the eight papers showed a negative effect of obesity on the healing after non-surgical periodontal therapy. Bouaziz et al. [28] revealed that normal-weight patients had a better response to periodontal treatment than obese patients. This effect was specially observed for moderate-to-deep pockets. This fact suggests that the more severe the periodontitis the more pronounced is the negative effect of obesity on periodontal treatment outcome. They showed in the multivariate analysis that obesity was significantly associated with percentage changes of $\mathrm{PD}>$ $5 \mathrm{~mm}$ and numbers of improving sites $(\mathrm{p} \leq 0.05)$. In the univariate analysis all periodontal parameters improve more in patients suffering from more severe periodontitis at baseline. Other patient characteristics, like age, sex, obesity, and WHR, were not associated with periodontal parameter changes. Gonçalves et al. [31] showed that patients with obesity and chronic periodontitis had a lower PDD reduction than patients without obesity. The measurement of the 
Table 7 Non-surgical periodontal treatment, treatment time and limitations

\begin{tabular}{lll}
\hline Author \& Year & Non-surgical periodontal treatment & $\begin{array}{l}\text { Treatment time; } \\
\text { periodontal maintenance }\end{array}$ \\
Al - Zahrani & Oral hygiene instructions, & n.r.; \\
et al. 2012 [8] & SRP performed by one operator & n.r. \\
Bouaziz et al. & Oral hygiene instructions (brushing & $2-3$ sessions for probing depth (PD) > \\
2015 [28] & technique: Bass technique, use of & $5 \mathrm{~mm}$ lasting $1 \mathrm{~h}$ each within 7 days; \\
& interproximal hygiene devices, CHX & Oral hygiene was controlled at each \\
& mouthwash (0.12 \%) twice a day for & appointment, \\
& 7 days), & hygiene instructions were repeated if \\
& SRP under local anesthesia using an & needed \\
& ultrasonic device and manual curets, & at the 3-month examination, reinstruction \\
& aim of each session was to remove & of oral hygiene methods was performed if \\
& biofilms and calculus of scaled roots, & needed, and residual pockets $\geq 4$ mm were \\
& treatment protocol did not include & systematically scaled and planed
\end{tabular}

antibiotic treatment,

all treatments procedures were performed by the same periodontist (WB)

Duzagac et al. Oral hygiene instructions,

2015 [29] all kinds of dental treatments, such as fillings and adjustment of overhanging restorations, were completed immediately after patient enrollment,

SRP using a reduced Gracey curette set (Gracey, SAS 5-6, SAS 7-8, SAS 11-12, SAS13- 14, Hu-Friedy) with an ultrasonic device (Dentsply/Cavitron, SPS, 30 K, TFI 1000, Dentsply, York, PA, USA) under local anesthesia, endpoint of treatment: a smooth tooth surface, patients received no adjunctive therapy (local or systemic antibiotics, NSAl, laser, ozonotherapy and mouth rinses), an experienced periodontist (EC) performed all treatment procedures (SRP, providing oral hygiene instructions and organizing patient recalls)

Eldin et al. Oral hygiene instructions, 2013 [30] SRP

Gonçalves et al. Oral hygiene instructions, 2015 [31] Supragingival plaque and calculus removal, exodontia, filling overhang removal and provisional restoration as necessary, trained periodontist performed SRP with manual curets and ultrasonic device under local anesthesia, endpoint for each SRP appointment was "smoothness of the scaled roots."

Author \& Year Non-surgical periodontal treatment

Gonçalves et al. Oral hygiene instructions regarding 2015 [32]
SRP in 4 sequential visits with 2 or 3 days intervals completed within 12 days,

each appointment lasted 45 min; all patients received monthly periodontal maintenance, including oral hygiene reinforcement, scaling and polishing as necessary

n.r.; periodontal maintenance (re-examination) performed weekly for 3 months after therapy

4 to 6 appointments lasting $\approx 1$ h each, periodontal therapy was completed in 14 days;

periodontal maintenance at 3 and

6 months after therapy

Treatment time; periodontal maintenance

4 to 6 appointments lasting approximately $1 \mathrm{~h}$ each, Periodontal therapy completed within 2 weeks;

All patients received periodontal

maintenance

every 3 months post-therapy

\section{brushing technique and} use of dental floss,

supragingival plaque and calculus removal, exodontia, provisional restoration and filling overhang removal, as necessary, trained periodontist performed SRP using manual curettes (Hu-Friedy, Chicago, IL, USA) and ultrasonic device (Cavitron Select SPC, Dentsply professional, York, PA, USA), under local anaesthesia, without use of local and/or systemic antimicrobials.

\section{Limitations}

n.r.

During the study, two patients dropped out in each group for the following reasons:

1) two patients migrated;

2) one missed the 3-month examination;

3) one missed the final examination
8 were excluded for several reasons did not return for 3 months follow-up, pregnancy, weight loss n.r.

3 patients from the group without obesity and 6 from the group with obesity did not return for the 3-months follow-up visit and were excluded.

\section{Adverse events}

There were no patient and sampled site dropouts during the course of the study. 
Table 7 Non-surgical periodontal treatment, treatment time and limitations (Continued)

\begin{tabular}{|c|c|c|c|}
\hline $\begin{array}{l}\text { Lakkis et al. } \\
2011 \text { [33] }\end{array}$ & $\begin{array}{l}\text { Oral hygiene instructions, } \\
\text { SRP }\end{array}$ & $\begin{array}{l}\text { n.r.; } \\
\text { n.r. }\end{array}$ & n.r. \\
\hline $\begin{array}{l}\text { Suvan et al. } \\
2014[34]\end{array}$ & $\begin{array}{l}\text { Oral hygiene instructions, } \\
\text { full-mouth mechanical periodontal } \\
\text { debridement with hand and ultrasonic } \\
\text { instruments performed with local } \\
\text { anesthesia by the same clinician }\end{array}$ & $\begin{array}{l}\text { Within a 24-h period of time; } \\
\text { n.r. }\end{array}$ & $\begin{array}{l}\text { No individuals in the sample had missing } \\
\text { data for any of the covariates listed in this } \\
\text { study. }\end{array}$ \\
\hline
\end{tabular}

CHX chlorhexidine, min minute, $\mathrm{mm}$ millimeter, n.r. not reported, $P D$ pocket depth, SRP scaling and root planing

reduction in PPD $(\mathrm{mm})$ at full-mouth sites showed after 3 months a not statistically significant difference $(p=$ 0.08 ) between the obese group compared to the group without obesity. However after 6 months there was a statistically significant difference $(p=0.04)$. At this time point, especially deep sites ( $P P D \geq 7 \mathrm{~mm}$ ) showed a significantly better result in the group without obesity $(p=0.04)$. Another study of Gonçalves et al. [32] reported that patients with obesity had a significantly greater mean PD (6 months $p$-value $=0.04,12$ months $p$ value $=0.03$ ) than patients without obesity at six and 12 months post-therapy. The data of a study by Suvan et al. [34] corroborated these findings and showed that obesity was an independent impact value of poorer periodontal treatment outcome 2 months after therapy. The extent of the association between poorer periodontal treatment and obesity was similar to that of smoking $(p=0.02)$. They worked with 260 patients. This secondary analysis consisted of individuals participating in five clinical studies of non-surgical periodontal therapy over a 7year period. Lakkis et al. [33] selected 30 patients who were obese; 15 of them had previously undergone bariatric surgery, whereas the other half $(n=15)$ did not loose any weight and served as a control group. The bariatric surgery group reached a statistically significant greater mean PPD reduction $(0.45 \mathrm{~mm}$ versus $0.28 \mathrm{~mm})$ compared with the control (no surgery) group $(p=0.007$; Table 4).

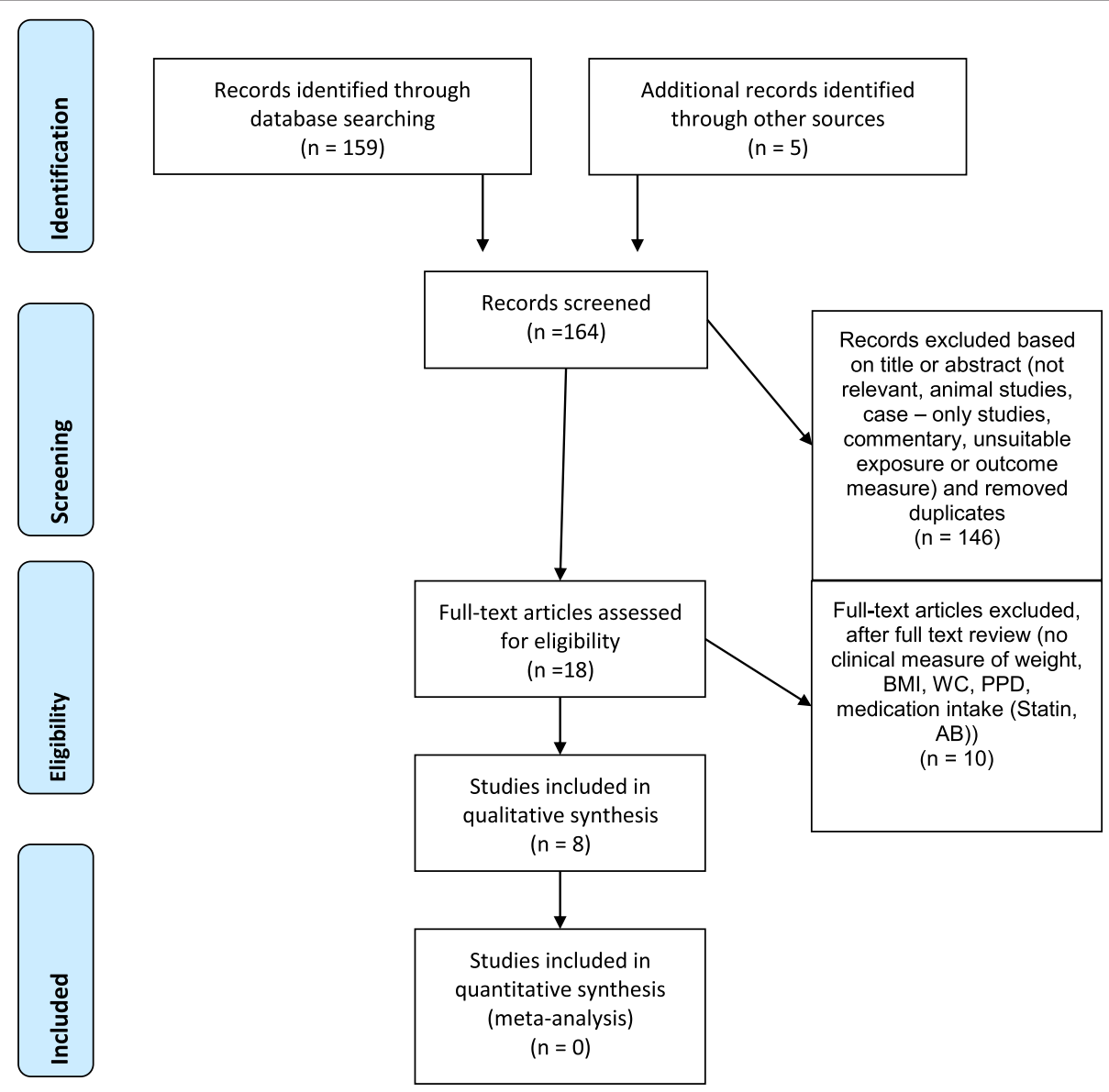

Fig. 1 PRISMA 2009 flow diagram 
Table 8 Newcastle - Ottawa Quality Assessment Scale

\begin{tabular}{|c|c|c|c|c|c|c|c|c|}
\hline & $\begin{array}{l}\text { Al - Zahrani } \\
\text { et al. } 2012 \\
\text { [8] }\end{array}$ & $\begin{array}{l}\text { Bouaziz et } \\
\text { al. } 2015 \\
{[28]}\end{array}$ & $\begin{array}{l}\text { Duzagac et al. } \\
2015[29]\end{array}$ & $\begin{array}{l}\text { Eldin et al. } 2013 \\
\text { [30] }\end{array}$ & $\begin{array}{l}\text { Gonçalves et al. } \\
2015 \text { [31] }\end{array}$ & $\begin{array}{l}\text { Gonçalves et al. } \\
2015[32]\end{array}$ & $\begin{array}{l}\text { Lakkis et al. } 2011 \\
\text { [33] }\end{array}$ & $\begin{array}{l}\text { Suvan et al. } 2014 \\
\text { [34] }\end{array}$ \\
\hline \multicolumn{9}{|l|}{ Selection } \\
\hline \multicolumn{9}{|l|}{ 1.) Is the case definition adequate? } \\
\hline $\begin{array}{l}\text { a) yes, with independent } \\
\text { validation * } \\
\text { b) yes, eg record linkage or } \\
\text { based on self reports } \\
\text { c) no description }\end{array}$ & * & * & * & * & * & * & * & \\
\hline \multicolumn{9}{|l|}{ 2) Representativeness of the cases } \\
\hline $\begin{array}{l}\text { a) consecutive or obviously } \\
\text { representative series of } \\
\text { cases * } \\
\text { b) potential for selection } \\
\text { biases or not stated }\end{array}$ & * & * & * & * & * & * & * & * \\
\hline \multicolumn{9}{|l|}{ 3) Selection of Controls } \\
\hline \multicolumn{9}{|l|}{$\begin{array}{l}\text { a) community controls } \\
\text { b) hospital controls } \\
\text { c) no description }\end{array}$} \\
\hline \multicolumn{9}{|l|}{ 4) Definition of Controls } \\
\hline \multicolumn{9}{|l|}{$\begin{array}{l}\text { a) no history of disease } \\
\text { (endpoint) * } \\
\text { b) no description of source }\end{array}$} \\
\hline \multicolumn{9}{|l|}{ Comparability } \\
\hline \multicolumn{9}{|l|}{$\begin{array}{l}\text { 1) Comparability of cases and controls on the } \\
\text { basis of the design or analysis }\end{array}$} \\
\hline $\begin{array}{l}\text { a) study controls for "smoking" * } \\
\text { b) study controls for "diabetes mellitus" * }\end{array}$ & *** & ** & ** & * & *** & *** & & * \\
\hline \multicolumn{9}{|l|}{ Exposure } \\
\hline \multicolumn{9}{|l|}{ 1)Ascertainment of exposure } \\
\hline \multicolumn{9}{|l|}{$\begin{array}{l}\text { a) secure record * } \\
\text { b) structured interview } \\
\text { where blind to case/control } \\
\text { status * } \\
\text { c) interview not blinded to } \\
\text { case/control status } \\
\text { d) written self report or } \\
\text { medical record only } \\
\text { e) no description }\end{array}$} \\
\hline \multicolumn{9}{|l|}{$\begin{array}{l}\text { 2) Same method of } \\
\text { ascertainment for cases and } \\
\text { controls }\end{array}$} \\
\hline $\begin{array}{l}\text { a) yes * } \\
\text { b) no }\end{array}$ & * & $*$ & * & * & * & * & & \\
\hline \multicolumn{9}{|l|}{ 3) Non-Response rate } \\
\hline $\begin{array}{l}\text { a) same rate for both } \\
\text { groups * } \\
\text { b) non respondents } \\
\text { described } \\
\text { c) rate different and no } \\
\text { designation }\end{array}$ & * & * & * & * & * & * & * & * \\
\hline$\overline{\text { Total }}$ & $6 \mathrm{x}$ 米 & $6 \mathrm{x} *$ & $6 \mathrm{x}$ 粠 & $5 \mathrm{x}$ 楼 & $6 x$ * & $6 \mathrm{x}$ 粦 & $3 \mathrm{x}$ 料 & 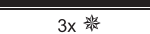 \\
\hline
\end{tabular}

Stars represent "awards" for each quality item and serve as a quick visual assessment. A maximum of one 'star' for each item within the 'Selection'/'Exposure' categories and a maximum of two 'stars' for 'Comparability' can be awarded

\section{Limitations}

Some patients could not be reviewed or discontinued the study for personal reasons [28, 29, 31]. Weight loss and pregnancy were additional reasons to be excluded in the study of Duzagac et al. [29] (Table 7).

The quality of the included studies was evaluated through the Newcastle-Ottawa Quality Assessment Scale (Table 8).

\section{Discussion}

This review focused on obesity and the outcome after non-surgical periodontal therapy and has shown that currently there is no really robust scientific evidence to reach solid conclusions and recommendations.
Three papers $[8,29,30]$ were found, which did not find any statistically significant negative impact of obesity on the response to non-surgical periodontal therapy, whereas five papers [28, 31-34] showed the opposite, i.e. a clearly negative influence of obesity on the treatment outcomes.

With regard to the quality of evidence, seven included papers [8, 28, 29, 31-34] reported some limitations.

Al-Zahrani et al. [8] included only women. Gonçalves and co-workers [31] did not consistently apply the accepted definitions of overweight and obesity, but rather included the waist-to-hip-ratio (WHR) for their definitions, probably leading to inclusion of patients with a BMI inferior to $30 \mathrm{~kg} / \mathrm{m}^{2}$ into the obesity group. Thus 
the results are difficult to interpret and compare with the other studies and are therefore not applicable for patients with a BMI $\geq 40 \mathrm{~kg} / \mathrm{m}^{2}$ [31]. Nonetheless, these studies still show a clearly better tendency with regard to the treatment response for patients without obesity as defined in their study. Lakkis et al. [33] chose another interesting way to find an impact of obesity on the outcome of non-surgical periodontal treatment. They compared obese people who had undergone bariatric surgery (BS) with obese who did not. After weight loss in the BS group, a reduction in total adipocytes might have resulted in a decrease in adipokines and pro-inflammatory mediators released by those adipose cells. This systemic inflammatory reduction might have played a role in reducing the insulin resistance resulting in a better outcome after periodontal therapy as suggested by the authors [33]. Some limitations in the specific profile of the obese patients (nondiabetic, non-smoker) in the paper of Bouaziz et al. (28) may restrict the extrapolation of the results to the whole obese population. Furthermore, the small sample size may also limit the power of this study. Duzagac et al. [29] failed to include a control group of periodontally healthy controls with obesity. Additionally the mean periodontitis parameters were within the limits of "moderate" periodontitis, and the WHR and BMI values of these obese patients were predominantly below those characterizing morbid obesity. So, the results of this study may not be extrapolated to those with severe periodontitis or morbid obesity. The second included study of Gonçalves and co-workers [32] assumes that the high interpatient variance in adipokine levels may reduce the statistical power to detect treatment effects, as previously reported. The results presented by Suvan et al. [34] may have been influenced by study limitations linked with unequal numbers in BMI categories and sample size. In addition, there may have been limitations with regard to the interpretation associated with the post hoc secondary analysis experimental design, although variation in clinical assessment and treatment was minimized by examiner and treatment clinician stability. This study did not constitute a higher level of evidence in the context of evidencebased health care levels of scientific evidence [34].

Overall, obesity is an obvious, visible stigma so that the studies cannot be considered blinded. This may be another possible bias in each of the studies.

Five studies $[8,28,29,31,32]$ excluded smokers. Since smoking influences periodontal health these studies are biased [9] and may not be fully representative for the typical overall population. Overall it appears, however, that a positive effect of normal weight is present in nonsmokers [28, 31, 32] and in smokers [34].

As mentioned before, the included studies differed in statistical methods, populations, sample sizes, definition of chronic periodontitis, definition of obesity, time of outcome measurement, smoking status, periodontal assessment and non-surgical periodontal therapy. Therefore, it was not only impossible to perform a meta-analysis but also draw clear conclusions.

Nonetheless there is a consensus in the studies that obesity is associated with different baseline PPD levels. The large cohort in the study of Suvan et al. [34] and the long term results of Gonçalves et al. [31, 32] may lead to the conclusion that obesity is an important negative factor which influences non-surgical periodontal therapy.

In summary, all studies $[8,28,29,31-34]$ included in this review validated the efficacy of non-surgical periodontal therapy, except the study of Eldin and co-workers [30] who did not report any efficacy of the therapy (Table 3). Clinically, it appears obvious that a therapy is necessary to reach periodontal health independent of the patient's body mass index.

Because this systematic review provided only moderate evidence that obesity is an important factor for nonsurgical periodontal therapy, future prospective cohort studies are needed to confirm these findings [36]. Such trials should be of high methodological quality. They should control important confounding factors such as smoking status, severity of chronic periodontitis, severity of obesity. Every patient should get the same periodontal treatment and periodontal maintenance. Overall, there is a possibility to solve the research question even though blinding of the examiners to obesity or non-obesity status is not practically possible.

Clinicians should know that obesity may have some influence on periodontal status and are likely to have a negative impact on the clinical outcome of conservative treatment, even if this systematic review found only five $[28,31-34]$ out of eight papers [8, 28-34] corroborating the influence of obesity on the clinical periodontal outcome focusing on PPD as surrogate parameter for periodontal healing.

Clinicians might consider a weight reduction diet as an additional treatment for periodontal health with a positive effect expected after 6 and 12 months [31, 32]. Also it should not be neglected that weight control has substantial other beneficial health effects which on their own justify such a recommendation.

\section{Conclusion}

This systematic review indicates a possible negative relationship between obesity and poorer treatment outcome in obese patients after non-surgical therapy based on the results of five out of eight studies. Three of these studies denied an impact of obesity on the treatment. The potentially inferior healing response could be based on pathophysiological inflammatory models.

Baseline levels showed also a poorer periodontal health in patients with obesity compared with non-obese patients. 
No study found any better dental health parameters in obese than in non-obese individuals, and although dental health may not be the most important target for arriving at a near normal body weight, a person who can keep his or her body weight near normal might, in addition to all other established health benefits, count on having better periodontal health than if they are obese.

\section{Acknowledgment}

The authors would like to thank Mrs. Dr. Martina Gosteli, librarian of the main library of the University of Zurich who performed the electrical literature search. This study was supported by the Clinic of Preventive Dentistry, Periodontology and Cariology (Center of Dental Medicine) of the University of Zurich.

\section{Authors' contributions}

FAG and PRS conceived the study, participated in its design, did the literature search and drafted the manuscript. PS helped to supervise the methodological correctness of the performed study and the coordination. OAS and JHB provided the required medical theoretical background for this study and participated in the study design. $\mathrm{CH}$ helped with the statistical evaluation of the papers and the tables. All authors carefully read and approved the final text.

\section{Competing interest}

The authors declare that they have no competing interests.

\section{Author details}

${ }^{1}$ Clinic of Preventive Dentistry, Periodontology and Cariology, Center of Dental Medicine, University of Zurich, Plattenstrasse 11, CH-8032 Zurich, Switzerland. 'Department of Internal Medicine, Cantonal Hospital Baden, Baden, Switzerland. ${ }^{3}$ Department of Statistics, Ludwig-Maximilians-University of Munich, Munich, Germany.

\section{Received: 28 January 2016 Accepted: 11 August 2016}

\section{Published online: 02 September 2016}

\section{References}

1. Saito T, Shimazaki Y, Koga T, Tsuzuki M, Ohshima A. Relationship between upper body obesity and periodontitis. J Dent Res. 2001;80:1631-6.

2. Pischon N, Heng N, Bernimoulin JP, Kleber BM, Willich SN, Pischon T. Obesity, inflammation, and periodontal disease. J Dent Res. 2007;86:400-9.

3. Francesco Branca HNuTL. Die Herausforderung Adipositas und Strategien zu ihrer Bekämpfung in der Europäischen Region der WHO. 2007.

4. Suresh S, Mahendra J. Multifactorial relationship of obesity and periodontal disease. J Clin Diagn Res. 2014;8:ZE01-03.

5. Wang Y, Rimm EB, Stampfer MJ, Willett WC, Hu FB. Comparison of abdominal adiposity and overall obesity in predicting risk of type 2 diabetes among men. Am J Clin Nutr. 2005;81:555-63.

6. Yusuf S, Hawken S, Ounpuu S, Bautista L, Franzosi MG, Commerford P, et al. Obesity and the risk of myocardial infarction in 27,000 participants from 52 countries: a case-control study. Lancet. 2005;366:1640-9.

7. Słotwińska S. Host response, obesity, and oral health. Cent Eur J Immunol. 2015;40:201-5

8. Al-Zahrani MS, Alghamdi HS. Effect of periodontal treatment on serum C- reactive protein level in obese and normal-weight women affected with chronic periodontitis. Saudi Med J. 2012;33:309-14.

9. Nishida NTM, Hayashi N, Nagata H, Takeshita T, Nakayama K, et al. Determination of smoking and obesity as periodontitis risks using the classification and regression tree method. J Periodontol. 2005:76:923-8.

10. Perlstein MI, Bissada NF. Influence of obesity and hypertension on the severity of periodontitis in rats. Oral Surg Oral Med Oral Pathol. 1977:43:707-19.

11. Koletsky S. Obese Spontaneously Hypertensive Rats-A Model for Study of Atherosclerosis. Exp Mol Pathol. 1973:19:53-60.

12. Chaffee BW, Weston SJ. Association between chronic periodontal disease and obesity: a systematic review and meta-analysis. J Periodontol. 2010;81:1708-24
13. Wimmer GPB. A critical assessment of adverse pregnancy outcome and periodontal disease. J Clin Periodontol. 2008:35:380-97.

14. Blaizot A, Vergnes JN, Nuwwareh S, Amar J, Sixou M. Periodontal diseases and cardiovascular events: metaanalysis of observational studies. Int Dent J. 2009;59:197-209.

15. Tomar SL, Asma S. Smoking-attributable periodontitis in the United States: findings from NHANES III. National Health and Nutrition Examination Survey. 2000;71:743-51.

16. Tonetti MS. Cigarette smoking and periodontal diseases: etiology and management of disease. Ann Periodontol. 1998;1:88-101.

17. Arnaud Chiolero DF, Fred P, Jacques C. Consequences of smoking for body weight, body fat distribution, and insulin resistance. Am J Clin Nutr. 2008:87:801-9.

18. Fei Xu, BEcon, Xiao-Mei Yin BM, Youfa Wang. The association between amount of cigarettes smoked and overweight, central obesity among Chinese adults in Nanjing, China. Asia Pac J Clin Nutr. 2007;16:240-247.

19. Hou X, Jia W, Bao Y, Lu H, Jiang S, Gu H, Xiang K. Risk factors for overweight and obesity, and changes in body mass index of Chinese adults in Shanghai. BMC Public Health. 2008;8:389.

20. Altay U, Gurgan CA, Agbaht K. Changes in inflammatory and metabolic parameters after periodontal treatment in patients with and without obesity. J Periodontol. 2013;84:13-23.

21. David Moher AL, Tetzlaff J, Altman DG. Preferred Reporting Items for Systematic Reviews and Meta-Analyses: The PRISMA Statement. J Clin Epidemiol. 2009;62:1006-12.

22. Buset SL, Zitzmann NU, Weiger R, Walter C. Non-surgical periodontal therapy supplemented with systemically administered azithromycin: a systematic review of RCTs. Clin Oral Investig. 2015;19(8):1763-75.

23. D'Aiuto F, Nibali L, Parkar M, Suvan J, Tonetti MS. Short-term effects of intensive periodontal therapy on serum inflammatory markers and cholesterol. J Dent Res. 2005;84:269-73.

24. Duan JY, O-YX, Zhou YX. Effect of periodontal initial therapy on the serum level of lipid in the patients with both periodontitis and hyperlipidemia. Bejing Da Xue Xue Bao. 2009:41:36-39.

25. Iwamoto Y, Nishimura F, Soga Y, Takeuchi K, Kurihara M, Takashiba S, et al. Antimicrobial periodontal treatment decreases serum C-reactive protein, tumor necrosis factor-alpha, but not adiponectin levels in patients with chronic periodontitis. J Periodontol. 2003;74:1231-6.

26. Kardeşler BN, Cetinkalp S, Kinane DF. Adipokines and inflammatory mediators after initial periodontal treatment in patients with type 2 diabetes and chronic periodontitis. J Periodontol. 2010;81:24-33.

27. Tandon S, Lamba AK, Verma M, Munjal A, Faraz F. Effect of Periodontal Therapy on Serum Lipid Levels. Indian J Med. 2010;1:19-25.

28. Bouaziz W, Davideau JL, Tenenbaum H, Huck O. Adiposity Measurements and Non-Surgical Periodontal Therapy Outcomes. J Periodontol. 2015;86:1030-7.

29. Duzagac E, Cifcibasi E, Erdem MG, Karabey V, Kasali K, Badur S, et al. Is obesity associated with healing after non-surgical periodontal therapy? A local vs. systemic evaluation. J Periodontal Res. 2015. [Epub ahead of print]

30. Eldin AM, Nasr SA, Hassan NE. Effect of non-surgical periodontal therapy on interleukin-8(il-8) level in gingival crevicular fluid in overweight and obese subjects with chronic periodontitis. World J Med Sci. 2013:9:173-9.

31. Goncalves TE, Feres M, Zimmermann GS, Faveri M, Figueiredo LC, Braga PG, et al. Effects of scaling and root planing on clinical response and serum levels of adipocytokines in patients with obesity and chronic periodontitis. J Periodontol. 2015:86:53-61.

32. Goncalves TE, Zimmermann GS, Figueiredo LC, Souza Mde C, da Cruz DF, Bastos MF, et al. Local and serum levels of adipokines in patients with obesity after periodontal therapy: one-year follow-up. J Clin Periodontol. 2015;42:431-9.

33. Lakkis D, Bissada NF, Saber A, Khaitan L, Palomo L, Narendran S, et al. Response to periodontal therapy in patients who had weight loss after bariatric surgery and obese counterparts: a pilot study. J Periodontol. 2012;83:684-9.

34. Suvan J, Petrie A, Moles DR, Nibali L, Patel K, Darbar U, et al. Body mass index as a predictive factor of periodontal therapy outcomes. J Dent Res. 2014;93:49-54

35. Genco RJ, Ho A, Nishimura F, Murayama Y. A proposed model linking inflammation to obesity, diabetes, and periodontal infections. J Periodontol. 2005;76:2075-84. 
36. Sgolastra F, Severino M, Pietropaoli D, Gatto R, Monaco A. Effectiveness of Periodontal Treatment to Improve Metabolic Control in Patients With Chronic Periodontitis and Type 2 Diabetes: A Meta-Analysis of Randomized Clinical Trials. J Periodontol. 2013:84:958-73.

37. Europerio 4, Berlin, Germany, 19-21 June 2003. J Clin Periodontol. 2003;30:5-100

38. Abstracts for the Royal Australian and New Zealand College of Psychiatrists 40th Congress. Psychiatry in a Changing World, Sydney, Australia, Sunday 22-Thursday 26 May, 2005. Aust N Z J Psychiatry. 2005;39:A97-186.

39. 5th Joint Meeting of the European Tissue Repair Society and the Wound Healing Society. Wound Repair Regen. 2009;17:A54-87.

40. Recently published abstracts. Altern Med Rev. 2010;15:369-80.

41. DENTSPLY. Posters. J Dent Hyg. 2012;86:322-6.

42. HealthBeat. Dent Assist. 2007;76:59-63. 55p.

43. Abstracts for Poster Presentations. 2nd North American/ Global Dental Hygiene Research Conference Bethesda, MD, October 20-22, 2011. J Dent Hyg. 2012;86:39-53. 15p.

44. Abstracts for the International Symposium on Dental Hygiene, Cape Town, South Africa, August 14-17, 2013. Int J Dent Hyg. 2013;11:156-73.

45. Abou Sulaiman AE, Shehadeh RMH. Assessment of Total Antioxidant Capacity and the Use of Vitamin C in the Treatment of Non-Smokers With Chronic Periodontitis. J Periodontol. 2010;81:1547-54.

46. Acharya A, Bhavsar N, Jadav B, Parikh H. Cardioprotective effect of periodontal therapy in metabolic syndrome: a pilot study in Indian subjects. Metab. 2010;8:335-41.

47. Akpinar A, Toker $\mathrm{H}$, Ozdemir $\mathrm{H}$, Bostanci $\mathrm{V}$, Aydin $\mathrm{H}$. The effects of non-surgical periodontal therapy on oxidant and anti-oxidant status in smokers with chronic periodontitis. Arch Oral Biol. 2013;58:717-23.

48. Armstrong BL, Sensat ML, Stoltenberg JL. Halitosis: a review of current literature. J Dent Hyg. 2010;84:65-74.

49. Arora N, Avula H, Avula JK. The adjunctive use of systemic antioxidant therapy (lycopene) in nonsurgical treatment of chronic periodontitis: A short-term evaluation. Quintessence Int. 2013;44:399-409.

50. Basegmez C, Berber L, Yalcin F. Clinical and Biochemical Efficacy of Minocycline in Nonsurgical Periodontal Therapy: A Randomized Controlled Pilot Study. J Clin Pharmacol. 2011;51:915-22.

51. Bresolin AC, Pronsatti MM, Pasqualotto LN, Nassar PO, Jorge AS, da Silva EAA, et al. Lipid profiles and inflammatory markers after periodontal treatment in children with congenital heart disease and at risk for atherosclerosis. Vasc Health Risk Manage. 2013;9:703-9.

52. Bresolin AC, Pronsatti MM, Pasqualotto LN, Nassar PO, Jorge AS, Da Silva EAA, et al. Effectiveness of periodontal treatment on the improvement of inflammatory markers in children. Arch Oral Biol. 2014;59:639-44.

53. Caspersen CJ, Thomas GD, Boseman LA, Beckles GLA, Albright AL. Aging, Diabetes, and the Public Health System in the United States. Am J Public Health. 2012;102:1482-97.

54. Caula AL, Lira-Junior R, Tinoco EM, Fischer RG. The effect of periodontal therapy on cardiovascular risk markers: a 6-month randomized clinical trial. J Clin Periodontol. 2014;41:875-82.

55. Chapple ILC. Oxidative stress, nutrition and neutrogenomics in periodontal health and disease. Int J Dent Hyg. 2006;4:15-21.

56. Chapple ILC, Brock GR, Milward MR, Ling N, Matthews JB. Compromised GCF total antioxidant capacity in periodontitis: cause or effect? J Clin Periodontol. 2007;34:103-10.

57. Chandni R, Mammen J, Joseraj MG, Joseph R. Effect of Nonsurgical Periodontal Therapy on Insulin Resistance in Patients with Type 2 Diabetes Mellitus and Chronic Periodontitis. Diabetes. 2015;64:A692.

58. Chaston R, Sabatini R, Koertge TE, Brooks CN, Schenkein HA. Serum anticardiolipin concentrations in patients with chronic periodontitis following scaling and root planing. J Periodontol. 2014;85:683-7.

59. Chee HK, Lim LP, Tay F, Thai AC, Sum CF. Non-surgical periodontal treatment and lipid levels in diabetic patients. Ann R Australas Coll Dent Surg. 2008;19:183.

60. Chee B, Park B, Bartold P. Periodontitis and type II diabetes: a two-way relationship. Int J Evid-Based Healthcare. 2013;11:317-29. 313p.

61. Chen L, Luo G, Xuan D, Wei B, Liu F, Li J, et al. Effects of non-surgical periodontal treatment on clinical response, serum inflammatory parameters, and metabolic control in patients with type 2 diabetes: a randomized study. J Periodontol. 2012;83:435-43.

62. D'Aiuto F, Parkar M, Andreou G, Brett PM, Ready D, Tonetti MS. Periodontitis and atherogenesis: causal association or simple coincidence? A pilot intervention study. J Clin Periodontol. 2004;31:402-11.
63. D'Aiuto F, Ready D, Tonetti MS. Periodontal disease and C-reactive protein-associated cardiovascular risk. J Periodontal Res. 2004;39:236-41.

64. D'Aiuto F, Parkar M, Nibali L, Suvan J, Lessem J, Tonetti MS. Periodontal infections cause changes in traditional and novel cardiovascular risk factors: results from a randomized controlled clinical trial. Am Heart J. 2006;151:977-84.

65. Deppe H, Hohlweg-Majert B, Holzle F, Schneider KT, Wagenpfeil S. Pilot study for periodontal treatment and pregnancy outcome: a clinical prospective study. Quintessence Int. 2010;41:e101-110.

66. Dodington DW, Fritz PC, Sullivan PJ, Ward WE. Higher Intakes of Fruits and Vegetables, beta-Carotene, Vitamin C, alpha-Tocopherol, EPA, and DHA Are Positively Associated with Periodontal Healing after Nonsurgical Periodontal Therapy in Nonsmokers but Not in Smokers. Jutr. 2015;145:2512-9.

67. Draper C. Advances in technology and periodontal therapy. J Calif Dent Hyg Assoc. 2010;25:12-4.

68. Edwards J. Exploring the link between oral health \& systemic disease. Access. 2006:20:15-20.

69. Efurd MG, Bray KK, Mitchell TV, Williams K. Comparing the Risk Identification and Management Behaviors between Oral Health Providers for Patients with Diabetes. J Dent Hyg. 2012;86:130-40.

70. Elliott-Smith S. Periodontal Disease: A Patient-by-Patient Approach. Access. 2011;25:28-32.

71. Engebretson SP, Hyman LG, Michalowicz BS. Hemoglobin a1c levels among patients with diabetes receiving nonsurgical periodontal treatment - Reply. JAMA. 2014;311:1921-2.

72. Fairfield C. Photodisinfection - innovative adjuntive therapy. J Calif Dent Hyg Assoc. 2010;25:20-2.

73. Fang F, Wu B, Qu Q, Gao J, Yan W, Huang X, et al. The clinical response and systemic effects of non-surgical periodontal therapy in end-stage renal disease patients: a 6-month randomized controlled clinical trial. J Clin Periodontol. 2015;42:537-46.

74. Fentoglu O, Sozen T, Oz SG, Kale B, Sonmez Y, Tonguc MO, et al. Short-term effects of periodontal therapy as an adjunct to anti-lipemic treatment. Oral Dis. 2010;16:648-54

75. Fine JB. The influence of periodontal inflammation on systemic diseases and medical conditions. Access. 2007:21:14-9.

76. Fokkema SJ. Peripheral blood monocyte responses in periodontitis. Int J Dent Hyg. 2012;10:229-35.

77. Fu YW, Li XX, Xu HZ, Gong YQ, Yang Y. Effects of periodontal therapy on serum lipid profile and proinflammatory cytokines in patients with hyperlipidemia: a randomized controlled trial. Clin Oral Investig. 2015;20: 1263-9.

78. Garcia VG, Takano RY, Fernandes LA, de Almeida JM, Theodoro LH. Treatment of experimental periodontal disease by a selective inhibitor of cyclooxygenase-2 with scaling and root planing (SRP). Inflammopharmacology. 2010;18:293-301.

79. Giblin LJ, Boyd LD, Rainchuso L, Chadbourne D. Short-term effects of non-surgical periodontal therapy on clinical measures of impaired glucose tolerance in people with prediabetes and chronic periodontitis. J Dent Hyg. 2014;88 Suppl 1:23-30

80. Gluch Jl. Commentary on "The effect of periodontal therapy on TNF-alpha IL-6 and metabolic control in type 2 diabetics". Access. 2007;21:26-8.

81. Goldie MP. Perio trends. Dispelling myths about periodontal disease. Access. 2002;16:40.

82. Goldie MP. C-reactive protein, cardiovascular disease, and periodontal disease. Int J Dent Hyg. 2004;2:139-41.

83. Goldie MP. What is new in research? Antioxidants in oral health care: making the connection. Int J Dent Hyg. 2005;3:93-5.

84. Griffin SO, Jones JA, Brunson D, Griffin PM, Bailey WD. Burden of Oral Disease Among Older Adults and Implications for Public Health Priorities. Am J Public Health. 2012;102:411-8.

85. Gurenlian JR. Inflammation: the relationship between oral health and systemic disease. Access. 2006;20:1-9. 9p.

86. Gurenlian JR. Inflammation: the relationship between oral health and systemic disease. Dent Assist. 2009;78:8.

87. Hammaker BG. Pharmacologic interventions for controlling the inflammatory cascade in periodontal disease. Access. 2010;24:19.

88. Horwitz J, Hirsh I, Machtei EE. Oral aspects of Gaucher's disease: a literature review and case report. J Periodontol. 2007;78:783-8. 
89. Hovliaras-Delozier CA. A common voice. Access. 2008;22:22-9.

90. Ide M, Jagdev D, Coward PY, Crook M, Barclay GR, Wilson RF. The short-term effects of treatment of chronic periodontitis on circulating levels of endotoxin, C-reactive protein, tumor necrosis factor-alpha, and interleukin-6. J Periodontol. 2004;75:420-8.

91. Ide M. Intensive periodontal treatment including extractions is associated with more immediate systemic inflammation but improved longer-term endothelial function compared to simple scaling. J Evid Based Dent Pract. 2007;7:162-4. 163p.

92. Jahn C. Diabetes and periodontal health. Dent Assist. 2004;73:24-9.

93. Jahn C. STANDARDS. Standard 1: Assessment: III. Risk Assessment. Access. 2015;29:19-23. 15p.

94. Jaiswal GR, Jain VK, Dhodapkar SV, Kumathalli KI, Kumar R, Nemawat $A$, et al. Impact of bariatric surgery and diet modification on periodontal status: A six month cohort study. J Clin Diagn Res. 2015;9:ZC43-5.

95. Janket SJ. Scaling and root-planing (SRP) may improve glycemic control and lipid profile in patients with chronic periodontitis (CP) and type 2 diabetes (DM2) in a specific subgroup: a meta-analysis of randomized clinical trials. J Evid Based Dent Pract. 2014;14:31-3.

96. Jared $\mathrm{H}$, Boggess KA. Periodontal diseases and adverse pregnancy outcomes: a review of the evidence and implications for clinical practice. J Dent Hyg. 2008; 82:24-41.

97. Jiang $H$, Xiong $X$, Su $Y$, Zhang $Y$, Wu H, Jiang $Z$, et al. A randomized controlled trial of pre-conception treatment for periodontal disease to improve periodontal status during pregnancy and birth outcomes. BMC Pregnancy Childbirth. 2013;13:228.

98. Kamil W, Al Habashneh R, Khader Y, Al Bayati L, Taani D. Effects of nonsurgical periodontal therapy on $C$-reactive protein and serum lipids in Jordanian adults with advanced periodontitis. J Periodontal Res. 2011;46:616-21.

99. Kamilov KP. Relationship between chronic periodontitis clinical manifestation and lipid peroxidation in saliva. Uzbekiston Tibbiet Zhurnali. 1998;0:37-9

100. Kapellas K, Do LG, Mark Bartold P, Skilton MR, Maple-Brown LJ, O'Dea K, et al. Effects of full-mouth scaling on the periodontal health of Indigenous Australians: a randomized controlled trial. J Clin Periodontol. 2013:40:1016-24.

101. Keller A, Rohde JF, Raymond K, Heitmann BL. Association between periodontal disease and overweight and obesity: a systematic review. J Periodontol. 2015;86:766-76.

102. Kiany F, Hedayati A. Evaluation of serum anti-cardiolipin antibodies after non-surgical periodontal treatment in chronic periodontitis patients. Odontology. 2014;103:203-9.

103. Kiany F, Hedayati A. Evaluation of serum anti-cardiolipin antibodies after non-surgical periodontal treatment in chronic periodontitis patients. Odontology. 2015;103:203-9.

104. Kipp A, Majeski J. New trends in perio. Access. 2003;17:10-5.

105. Kudva P, Tabasum ST, Garg N. Evaluation of clinical and metabolic changes after non surgical periodontal treatment of type 2 diabetes mellitus patients: A clinico biochemical study. J Indian Soc Periodontol. 2010;14:257-62.

106. Kumar M, Bandyopadhyay P, Mishra L, Das S, Kundu PK, Mistry S. Effect of periodontal therapy on glycemic control and circulating TNF-a in type 2 diabetic patients. Int J Diabetes Dev Ctries. 2015;35:96-102.

107. Kurti B, Tüter G, Serdar M, Pinar S, Demirel I, Toyman U. Gingival crevicular fluid prostaglandin $\mathrm{E}(2)$ and thiobarbituric acid reactive substance levels in smokers and non-smokers with chronic periodontitis following phase I periodontal therapy and adjunctive use of flurbiprofen. J Periodontol. 2007; 78:104-111.

108. Kurtis B, Tuter G, Serdar M, Pinar S, Demirel I, Toyman U. Gingival crevicular fluid prostaglandin E-2 and thiobarbituric acid reactive substance levels in smokers and non-smokers with chronic periodontitis following phase I periodontal therapy and adjunctive use of flurbiprofen. J Periodontol. 2007;78:104-11.

109. Lee HJ, Jun JK, Lee SM, Ha JE, Paik DI, Bae KH. Association between obesity and periodontitis in pregnant females. J Periodontol. 2014;85:e224-231.

110. Li X, Tse HF, Yiu KH, Zhang C, Jin LJ. Periodontal therapy decreases serum levels of adipocyte fatty acid-binding protein in systemically healthy subjects: a pilot clinical trial. J Periodontal Res. 2013:48:308-14.
111. Ling A, Wang P-W, Lin R-T, Hsieh C-J, Lee P-Y, Zhuang R-Y, et al. Evaluation of Periodontal Status and Effectiveness of Non-Surgical Treatment in Patients With Type 2 Diabetes Mellitus in Taiwan for a 1-Year Period. J Periodontol. 2012;83:621-8.

112. Lo JC, O'Ryan F, Yang J, Hararah MK, Gonzalez JR, Gordon N, et al. Oral Health Considerations in Older Women Receiving Oral Bisphosphonate Therapy. J Am Geriatr Soc. 2011;59:916-22. 917p.

113. Malhotra R, Kapoor A, Grover V, Grover D, Kaur A. Effect of Scaling and Root Planing on Erythrocyte Count, Hemoglobin and Hematocrit in Patients with Chronic Periodontal Disease. J Dent Hyg. 2012;86:195-203.

114. Mancl KA, Kirsner RS, Ajdic D. Wound biofilms: Lessons learned from oral biofilms. Wound Repair Regen. 2013;21:352-62.

115. Martinez GL, Koury JC, Brito F, Fischer RG, Gustafsson A, Figueredo CM. The impact of non-surgical periodontal treatment on serum levels of long chain-polyunsaturated fatty acids: a pilot randomized clinical trial. J Periodontal Res. 2014;49:268-74.

116. Martinez GL, Koury JC, Martins MA, Nogueira F, Fischer RG, Gustafsson A, et al. Serum level changes of long chain-polyunsaturated fatty acids in patients undergoing periodontal therapy combined with one year of omega-3 supplementation: a pilot randomized clinical trial. J Periodontal Implant Sci. 2014;44:169-77.

117. Matlock J, Ferguson K, Calef J, Abbott S. Obesity and Bariatric Surgery: Effects on the Oral Cavity. Access. 2012;26:8-11.

118. McDaniel JC, Roy S, Wilgus TA. Neutrophil activity in chronic venous leg ulcers-A target for therapy? Wound Repair Regen. 2013;21:339-51. 313p.

119. Meharwade W, Gayathri GV, Mehta DS. Effects of scaling and root planing with or without a local drug delivery system on the gingival crevicular fluid leptin level in chronic periodontitis patients: a clinico-biochemical study. J Periodontal Implant Sci. 2014;44:118-25.

120. Merchant AT. Hemoglobin A1c levels among patients with diabetes receiving nonsurgical periodontal treatment [1]. JAMA. 2014;311:1919.

121. Michalowicz BS, Hyman L, Wei H, Oates Jr TW, Reddy M, Paquette DW, et al. Factors associated with the clinical response to nonsurgical periodontal therapy in people with type 2 diabetes mellitus. JADA. 2014;145:1227-39. 1213p.

122. Mizrak T, Guncu GN, Caglayan F, Balci TA, Aktar GS, Ipek F. Effect of a controlled-release chlorhexidine chip on clinical and microbiological parameters and prostaglandin E-2 levels in gingival crevicular fluid. J Periodontol. 2006;77:437-43.

123. Moeintaghavi A, Arab HR, Bozorgnia Y, Kianoush K, Alizadeh M. Non-surgical periodontal therapy affects metabolic control in diabetics: a randomized controlled clinical trial. Aust Dent J. 2012;57:31-37.

124. Moravec $L$, Boyd LD. Bariatric Surgery and Implications for Oral Health: A Case Report. J Dent Hyg. 2011;85:166-76.

125. Muthu J, Muthanandam S, Mahendra J, Namasivayam A, John L, Logaranjini A. Effect of Nonsurgical Periodontal Therapy on the Glycaemic Control of Nondiabetic Periodontitis Patients: A Clinical Biochemical Study. Oral Health Prev Dent. 2015;13:261-6.

126. Nassar PO, Walker CS, Salvador CS, Felipetti FA, Orrico SRP, Nassar CA. Lipid profile of people with Diabetes mellitus type 2 and periodontal disease. Diabetes Res Clin Pract. 2012;96:35-9.

127. Newton KM, Chaudhari M, Barlow WE, Inge RE, Theis MK, Spangler LA, et al. A population-based study of periodontal care among those with and without diabetes. J Periodontol. 2011;82:1650-6.

128. Nichols FC, Levinbook H, Shnaydman M, Goldschmidt J. Prostaglandin E2 secretion from gingival fibroblasts treated with interleukin-1 beta: effects of lipid extracts from Porphyromonas gingivalis or calculus. J Periodontal Res. 2001;36:142-52.

129. Nielsen D, Walser C, Kodan G, Chaney RD, Yonkers T, VerSteeg JD, et al. Effects of treatment with clindamycin hydrochloride on progression of canine periodontal disease after ultrasonic scaling. Vet Ther. 2000;1:150-8.

130. Novakovic N, Cakic S, Todorovic T, Raicevic BA, Dozic I, Petrovic V, et al. Antioxidative Status of Saliva before and after Non-Surgical Periodontal Treatment. Srp Ark Celok Lek. 2013;141:163-8.

131. Novakovic N, Todorovic T, Rakic M, Milinkovic I, Dozic I, Jankovic S, et al. Salivary antioxidants as periodontal biomarkers in evaluation of tissue status and treatment outcome. J Periodontal Res. 2014;49:129-36. 
132. Oliveira AM, de Oliveira PA, Cota LO, Magalhaes CS, Moreira AN, Costa FO. Periodontal therapy and risk for adverse pregnancy outcomes. Clin Oral Investig. 2011;15:609-15.

133. Olsen NC. Health Access. 2006;20:56-7. 52p.

134. Paquette DW, Ryan ME, Wilder RS. Locally delivered antimicrobials: clinical evidence and relevance. J Dent Hyg. 2008;82:10-5.

135. Perayil J, Suresh N, Fenol A, Vyloppillil R, Bhaskar A, Menon S. Comparison of Hba1c Levels in Non-Diabetic Healthy Subjects and Subjects With Periodontitis Before and After Non-Surgical Periodontal Therapy. J Periodontol. 2014:85:1658-66.

136. Perayil J, Suresh N, Fenol A, Vyloppillil R, Bhaskar A, Menon S. Comparison of glycated hemoglobin levels in individuals without diabetes and with and without periodontitis before and after non-surgical periodontal therapy. J Periodontol. 2014;85:1658-66.

137. Phillips A. Oral complications of diabetes: an under-recognized condition. Pract Nurs. 2013;24:562-6.

138. Pradeep AR, Manjunath SG, Swati PP, Shikha C, Sujatha PB. Gingival crevicular fluid levels of leukotriene B4 in periodontal health and disease. J Periodontol. 2007;78:2325-30.

139. Price T. Periodontal disease and adverse pregnancy outcomes: treatment recommendations for the pregnant woman. Access. 2010;24:20-3.

140. Qiqiang L, Huanxin M, Xuejun G. Longitudinal study of volatile fatty acids in the gingival crevicular fluid of patients with periodontitis before and after nonsurgical therapy. J Periodontal Res. 2012;47:740-9.

141. Radafshar G, Torabi F, Mirfarhadi N. Short-term effects of intensive non-surgical periodontal therapy and low-dose doxycycline on serum levels of IL-6, TNF-alpha and lipid profile in advanced periodontitis. Afr J Microbiol Res. 2012;6:355-60.

142. Radnai M, Pal A, Novak T, Urban E, Eller J, Gorzo I. Benefits of periodontal therapy when preterm birth threatens. J Dent Res. 2009;88:280-4.

143. Raghavendra NM, Pradeep AR, Kathariya R, Sharma A, Rao NS, Naik SB. Effect of non surgical periodontal therapy on gingival crevicular fluid and serum visfatin concentration in periodontal health and disease. Dis Markers. 2012:32:383-8.

144. Ramirez JH, Arce RM, Contreras A. Periodontal treatment effects on endothelial function and cardiovascular disease biomarkers in subjects with chronic periodontitis: protocol for a randomized clinical trial. Trials. 2011;12:46.

145. Rasch MS, Mealey BL, Prihoda TJ, Woodard DS, McManus LM. The effect of initial periodontal therapy on salivary platelet-activating factor levels in chronic adult periodontitis. J Periodontol. 1995;66:613-23

146. Sadatmansouri S, Sedighpoor N, Aghaloo M. Effects of periodontal treatment phase I on birth term and birth weight. J Indian Soc Pedod Prev Dent. 2006;24:23-6.

147. Saffi MA, Furtado MV, Montenegro MM, Ribeiro IW, Kampits C, Rabelo-Silva $E R$, et al. The effect of periodontal therapy on C-reactive protein, endothelial function, lipids and proinflammatory biomarkers in patients with stable coronary artery disease: study protocol for a randomized controlled trial. Trials. 2013;14:283.

148. Williams KB. Effect of treating periodontal disease on cardiovascular markers. J Dent Hyg. 2007:81:49.

149. Sembene M, Moreau JC, Mbaye MM, Diallo A, Diallo PD, Ngom M, et al. Periodontal infection in pregnant women and low birth weight babies. Odontostomatol Trop. 2000;23:19-22.

150. Sengupta S, Fine J, Wu-Wang CY, Gordon J, Murty VLN, Slomiany A, et al. The relationship of prostaglandins to $C A M P, \lg G, \lg M$ and alpha-2macroglobulin in gingival crevicular fluid in chronic adult periodontitis. Arch Oral Biol. 1990:35:593-6.

151. Shimada $Y$, Komatsu $Y$, Ikezawa-Suzuki I, Tai H, Sugita N, Yoshie H. The effect of periodontal treatment on serum leptin, interleukin-6, and C-reactive protein. J Periodontol. 2010;81:1118-23.

152. Shimoe M, Yamamoto $T$, Iwamoto $Y$, Shiomi N, Maeda $H$, Nishimura F, et al. Chronic periodontitis with multiple risk factor syndrome: a case report. [Erratum appears in J Int Acad Periodontol. 2011 Oct;13(3):93]. J Int Acad Periodontol. 2011;13:40-7.

153. Singh N, Narula SC, Sharma RK, Tewari S, Sehgal PK. Vitamin E Supplementation, Superoxide Dismutase Status, and Outcome of Scaling and Root Planing in Patients With Chronic Periodontitis: A Randomized Clinical Trial. J Periodontol. 2014;85:242-9.
154. Siqueira MAD, Fischer RG, Pereira NR, Martins MA, Moss MB, Mendes Ribeiro AC, et al. Effects of non-surgical periodontal treatment on the L-arginine-nitric oxide pathway and oxidative status in platelets. Exp Biol Med. 2013;238:713-22.

155. Stewart JE, Wager KA, Friedlander AH, Zadeh HH. The effect of periodontal treatment on glycemic control in patients with type 2 diabetes mellitus. J Clin Periodontol. 2001;28:306-10.

156. Talbert J, Elter J, Jared HL, Offenbacher S, Southerland J, Wilder RS. The effect of periodontal therapy on TNF-alpha, IL-6 and metabolic control in type 2 diabetics. J Dent Hyg. 2006;80:7-7.

157. Tamaki N, Tomofuji T, Ekuni D, Yamanaka R, Yamamoto T, Morita M. Short-Term Effects of Non-Surgical Periodontal Treatment on Plasma Level of Reactive Oxygen Metabolites in Patients With Chronic Periodontitis. J Periodontol. 2009;80:901-6.

158. Tamaki N, Tomofuji T, Ekuni D, Yamanaka R, Morita M. Periodontal treatment decreases plasma oxidized LDL level and oxidative stress. Clin Oral Investig. 2011;15:953-8.

159. Tawfig A. Effects of non-surgical periodontal therapy on serum lipids and C-reactive protein among hyperlipidemic patients with chronic periodontitis. J Int Soc Prev Community Dent. 2015;5:S49-56.

160. Teles FR, Teles RP, Martin L, Socransky SS, Haffajee AD. Relationships among interleukin-6, tumor necrosis factor-alpha, adipokines, vitamin D, and chronic periodontitis. J Periodontol. 2012;83:1183-91.

161. Toker H, Akpinar A, Aydin H, Poyraz O. Influence of smoking on interleukin-1 beta level, oxidant status and antioxidant status in gingival crevicular fluid from chronic periodontitis patients before and after periodontal treatment. J Periodontal Res. 2012;47:572-7.

162. Tuter G, Kurtis B, Serdar M, Aykan T, Okyay K, Yucel A, et al. Effects of scaling and root planing and sub-antimicrobial dose doxycycline on oral and systemic biomarkers of disease in patients with both chronic periodontitis and coronary artery disease. J Clin Periodontol. 2007;34:673-81.

163. Vardar S, Baylas $H$, Huseyinov A. Effects of selective cyclooxygenase-2 inhibition on gingival tissue levels of prostaglandin E2 and prostaglandin F2alpha and clinical parameters of chronic periodontitis. J Periodontol. 2003;74:57-63.

164. Van Dyke TE, Hasturk H, Kantarci A, Freire MO, Nguyen D, Dalli J, et al. Proresolving nanomedicines activate bone regeneration in periodontitis. J Dent Res. 2015;94:148-56.

165. Vyas SP, Sihorkar V, Mishra V. Controlled and targeted drug delivery strategies towards intraperiodontal pocket diseases. J Clin Pharm Ther. 2000;25:21-42.

166. Wahid A, Chaudhry S, Ehsan A, Butt S, Khan AA. Bidirectional relationship between chronic kidney disease \& periodontal disease. Pak J Med Sci. 2013:29:211-5.

167. Wang XE, Xu L, Meng HX, Lü D, Chen ZB, Lu RF. Long-term clinical and hematologic effects of non-surgical treatment on aggressive periodontitis. Zhonghua Kou Qiang Yi Xue Za Zhi. 2013;48:467-71.

168. Wehmeyer MM, Kshirsagar AV, Barros SP, Beck JD, Moss KL, Preisser JS, et al. A randomized controlled trial of intensive periodontal therapy on metabolic and inflammatory markers in patients With ESRD: results of an exploratory study. Am J Kidney Dis. 2013;61:450-8.

169. Wei D, Zhang XL, Wang YZ, Yang CX, Chen G. Lipid peroxidation levels, total oxidant status and superoxide dismutase in serum, saliva and gingival crevicular fluid in chronic periodontitis patients before and after periodontal therapy. Aust Dent J. 2010;55:70-8.

170. Williams KB. Periodontal disease and type 2 diabetes. J Dent Hyg. 2009;83:8-44.

171. Williams KB, Bray KK. Increasing patient engagement in care: motivational i nterviewing. Access. 2009;23:36-9.

172. Wood N. Oral health - how to reduce risks of periodontitis. Posit Health. 2006:30-35.

173. Wu Y, Chen L, Wei B, Luo K, Yan FH. Effect of Non-Surgical Periodontal Treatment on Visfatin Concentrations in Serum and Gingival Crevicular Fluid of Patients With Chronic Periodontitis and Type 2 Diabetes Mellitus. J Periodontol. 2015:86:795-800.

174. Zare Javid A, Seal CJ, Heasman P, Moynihan PJ. Impact of a customised dietary intervention on antioxidant status, dietary intakes and periodontal indices in patients with adult periodontitis. J Hum Nutr Diet. 2014;27:523-32. 510p.

175. Zhou SY, Duan XQ, Hu R, Ouyang XY. Effect of non-surgical periodontal therapy on serum levels of TNF-a, IL-6 and C-reactive protein in periodontitis subjects with stable coronary heart disease. Chin J Dent Res. 2013;16:145-51 
176. Zuza EP, Barroso EM, Carrareto AL, Pires JR, Carlos IZ, Theodoro LH, et al. The role of obesity as a modifying factor in patients undergoing non-surgical periodontal therapy. J Periodontol. 2011;82:676-82.

177. Armitage GC. Periodontal diagnoses and classification of periodontal diseases. Periodontology 2000. 2004;34:9-21.

178. Silness J, Löe H. Periodontal Disease in Pregnancy II. Correlation between oral hygiene and periodontal condition. Acta Odontol Scand. 1964:22:121-35.

179. Löe HSJ. Periodontal disease in pregnancy. I. Prevalance and severity. Acta Odontol Scand. 1963;21:533-51.

Submit your next manuscript to BioMed Central and we will help you at every step:

- We accept pre-submission inquiries

- Our selector tool helps you to find the most relevant journal

- We provide round the clock customer support

- Convenient online submission

- Thorough peer review

- Inclusion in PubMed and all major indexing services

- Maximum visibility for your research

Submit your manuscript at www.biomedcentral.com/submit
Biomed Central 